\title{
Cost-Benefit Study of Culling Claim Folders at the Department of Veterans Affairs
}

by P. Charles, L.G. Hill, P. Johnson, and C.A. Riemer

Policy and Economic Analysis Group, Environmental Assessment and Information Sciences Division, Argonne National Laboratory, 9700 South Cass Avenue, Argonne, Illinois 60439

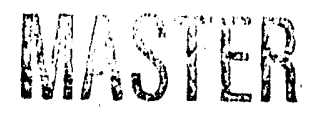

April 1990

Work sponsored by Department of Veterans Affairs, Velerans Benefits Administration 


\section{CONTENTS}

ACKNOWLEDGMENT $\ldots \ldots \ldots \ldots \ldots \ldots \ldots \ldots \ldots \ldots \ldots \ldots \ldots \ldots \ldots$

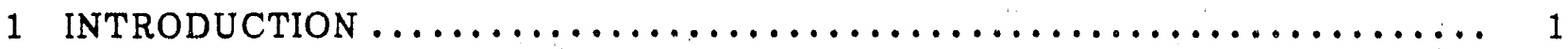

1.1 Objective of Study $\ldots \ldots \ldots \ldots \ldots \ldots \ldots \ldots \ldots \ldots \ldots \ldots \ldots \ldots \ldots \ldots \ldots \ldots$

1.1.1 Study of Culling Strategies $\ldots \ldots \ldots \ldots \ldots \ldots \ldots \ldots \ldots \ldots \ldots \ldots \ldots 1$

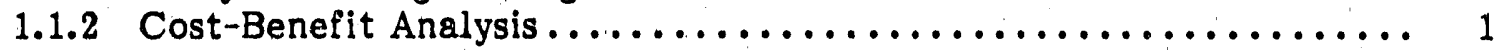

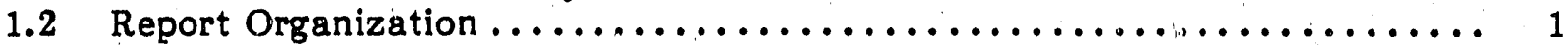

2 METHODOLOGY.................................... 2

2.1 Study of Culling Strategies $\ldots \ldots \ldots \ldots \ldots \ldots \ldots \ldots \ldots \ldots \ldots \ldots \ldots \ldots \ldots$

2.1 .1 Choosing Claim Folder Samples ..................... 2

2.1.2 Determining Folder Characteristics ................... 2

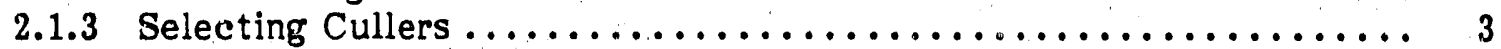

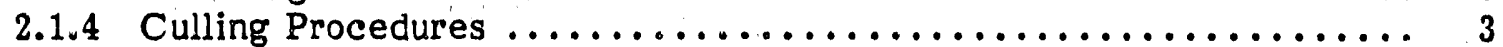

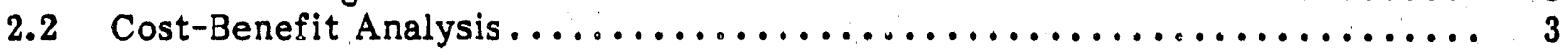

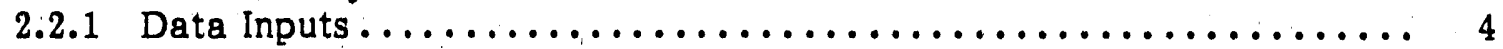

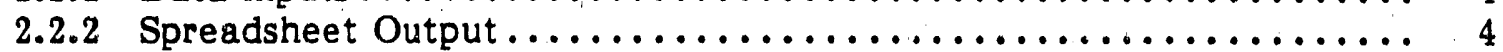

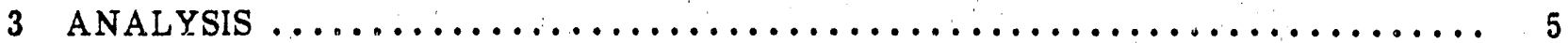

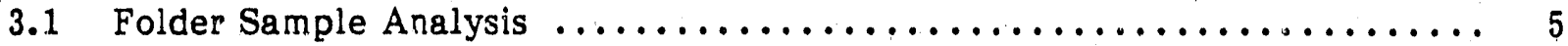

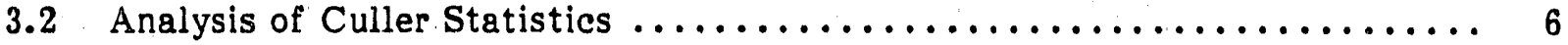

3.2.1 Documents Removed and Time Required ................ 6

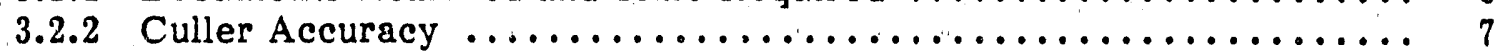

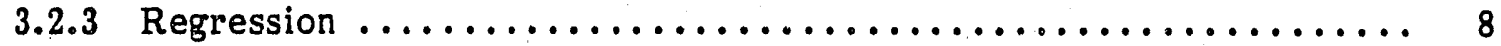

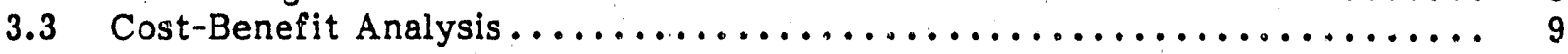

4 CONCLUSIONS $\ldots \ldots \ldots \ldots \ldots \ldots \ldots \ldots \ldots \ldots \ldots \ldots \ldots \ldots \ldots \ldots \ldots \ldots \ldots \ldots$

APPENDIX A: Cullers' Names and Government Service Levels .............. 13

APPENDIX B: Instructions to Cullers $\ldots \ldots \ldots \ldots \ldots \ldots \ldots \ldots \ldots \ldots \ldots \ldots \ldots$

APPENDIX C: Cost-Benefit Spreadsheets...................... 21

APPENDIX D: Spreadsheet on Claim Folder Thicknesses ............... 39

APPENDIX E: Statistics on Culling $\ldots \ldots \ldots \ldots \ldots \ldots \ldots \ldots \ldots \ldots \ldots \ldots \ldots \ldots$

APPENDIX F: Spreadsheet on Claim Folder Statistics by Culler ........... 47

APPENDIX G: List of Documents Removed During Culling $\ldots \ldots \ldots \ldots \ldots \ldots \ldots \ldots$

APPENDIX H: Calculation Tables $\ldots \ldots \ldots \ldots \ldots \ldots \ldots \ldots \ldots \ldots \ldots \ldots \ldots \ldots$

APPENDIX I: $\quad$ Regression Analysis $\ldots \ldots \ldots \ldots \ldots \ldots \ldots \ldots \ldots \ldots \ldots \ldots \ldots \ldots \ldots$ 


\section{TABLES}

$1 \quad$ Culler Accuracy $\ldots \ldots \ldots \ldots \ldots \ldots \ldots \ldots \ldots \ldots \ldots \ldots \ldots \ldots \ldots \ldots \ldots \ldots$

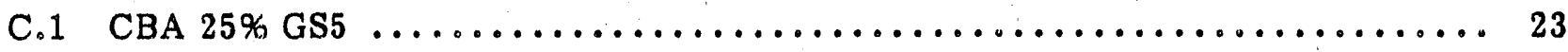

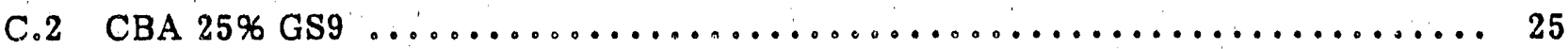

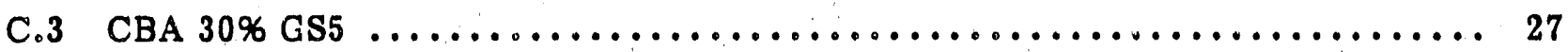

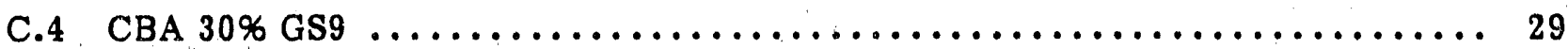

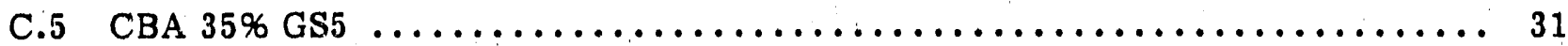

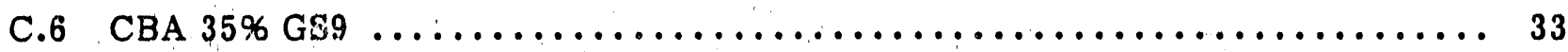

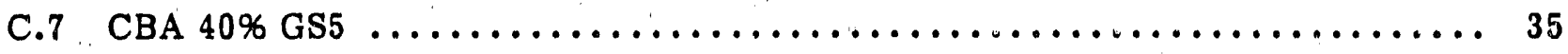

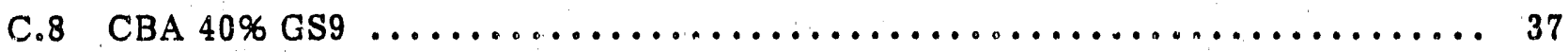

F.1 Folder Statisties for Eciwards $\ldots \ldots \ldots \ldots \ldots \ldots \ldots \ldots \ldots \ldots \ldots \ldots \ldots \ldots \ldots$

F.2 Folder Statistics for Rutledge $\ldots \ldots \ldots \ldots \ldots \ldots \ldots \ldots \ldots \ldots \ldots \ldots \ldots \ldots \ldots \ldots$

F.3 Folder Statistics for Stigler ............................ 51

F.4 Folder Statistics for Wheatley $\ldots \ldots \ldots \ldots \ldots \ldots \ldots \ldots \ldots \ldots \ldots \ldots \ldots \ldots \ldots \ldots$

F.5 Folder Statistics for Phillips $\ldots \ldots \ldots \ldots \ldots \ldots \ldots \ldots \ldots \ldots \ldots \ldots \ldots \ldots \ldots$

F.6 Folder Statistics for Wengerski.......................... 54

H.1 Calculations for Edwards $\ldots \ldots \ldots \ldots \ldots \ldots \ldots \ldots \ldots \ldots \ldots \ldots \ldots \ldots \ldots \ldots \ldots \ldots$

H.2 Calculations for Rutledge $\ldots \ldots \ldots \ldots \ldots \ldots \ldots \ldots \ldots \ldots \ldots \ldots \ldots \ldots \ldots \ldots \ldots \ldots \ldots \ldots$

H.3 Calculations for Stigler $\ldots \ldots \ldots \ldots \ldots \ldots \ldots \ldots \ldots \ldots \ldots \ldots \ldots \ldots \ldots \ldots \ldots$

H.4 Calculations for Wheatley $\ldots \ldots \ldots \ldots \ldots \ldots \ldots \ldots \ldots \ldots \ldots \ldots \ldots \ldots$

H.5 Calculations for Phillips $\ldots \ldots \ldots \ldots \ldots \ldots \ldots \ldots \ldots \ldots \ldots \ldots \ldots \ldots \ldots \ldots \ldots \ldots$

H.6 Calculations for Wengerski $\ldots \ldots \ldots \ldots \ldots \ldots \ldots \ldots \ldots \ldots \ldots \ldots \ldots \ldots \ldots \ldots$

I.1 R Squared Table $\ldots \ldots \ldots \ldots \ldots \ldots \ldots \ldots \ldots \ldots \ldots \ldots \ldots \ldots \ldots \ldots \ldots \ldots \ldots$

I.2 Regressions for Total File Thirkness Versus Total Culling Time .......... 74

I.3 Regressions for Total File Thickness Versus Total Percent Removed........ 74 


\section{ACKNOWLEDGMENT}

The authors would like to thank the staff members at the Department of Veterans Affairs (VA) Chicago Regional Office for their assistance and participation in both the pilot study and the cost-benefit study described in this report. These employees were very helpful and patient throughout the duration of the studies. We would also like to thank Steve Simmons from the VA's central office for his contribution and insights into the study. 


\title{
COST-BENEFIT STUDY OF THE CULLING OF CLAIM FOLDERS AT THE DEPARTMENT \\ OF VETERANS AFFAIRS
}

\author{
by \\ P. Charles, L.G. Hill, P. Johnson, and C.A. Riemer
}

\section{INTRODUCTION}

\subsection{OBJECTIVE OF STUDY}

Culling is the process of sorting through material to separate significant from insignificant information. Argonne National Laboratory (ANL) conducted a study on culling Veterans Benefits Administration (VBA) claim folders for two reasons. The first was to help the Department of Veterans Affairs (VA) learn about culling strategies that could be applied to veterans' claim folders located at the VA's regional offices (ROs) throughout the United States. The second was to show the VA the benefits that could be derived from culling.

\subsubsection{Study of Culling Strategies}

We looked at various culling itrategies that could be of benefit to the VA. Many aspects related to the culling process were studied, including folder thickness, time needed for culling, the type of documentation contained in each folder, the type of documentation retained or removed from each folder, and the degree of culling accuracy demonstrated by VA personnel.

\subsubsection{Cost-Benefit Analysis}

The benefit that the VA would incur from culling claim folders was analyzed. This cost-benefit analysis focused on benefits at each RO. It was based on the assumption that insignificant material would be culled from each claim folder and be either permanently discarded or sent to the St. Louis regional processing center (RPC) for storage. Rate of return, payback period, storage costs, and salaries were the variables considered.

\subsection{REPORT ORGANIZATION}

This report has four sections. Section 1 is the introduction. Section 2 discusses methodology; it describes the procedures used in both the culling process and the costbenefit analysis. The third section provides explanations and results from the analyses of file samples, culler statistics, and cost benefits. The final section, the conclusion, includes our recommendations on beneficial culling strategies that could be employed by the VA. 


\section{METHODOLOGY}

Our procedures for determining culling strategies were developed from data gathered in a pilot study we conducted at the VA's Chicago RO and from determinations we made about culling times, folder thicknesses, types of documents culled from claim folders, and culling accuracies. Our procedures and inputs for determining the cost benefits of eulling were developed in part from information in the RPC upgrade initiative provided by the VA. The cost-benefit analysis also considered the findings from the first part of this study (on strategies).

\subsection{STUDY OF CULLING STRATEGIES}

Our first step was to obtain a random sample of folders from the Chicago RO. The contents and size of each claim folder in the sample were analyzed. A group of VA personnel then culled a select number of these sample claim folders, and we recorded the results. Finally, the claim folders were restored to their original condition and returned to the Chicago RO's files.

\subsubsection{Choosing Claim Folder Samples}

On the basis of its pilot study, we determined that a random sample of 60 claim folders from the Chicago RO would be adequate to represent the characteristics of ail the claim folders at this office. Furthermore, because all VA ROs use the same claim folders, these 60 claim folders could also be used to represent the characteristics of claim folders at other ROs.

The random sample was chosen on the basis of a table of random numbers that listed potential samples. The first four digits of each number in the table were used. The first two digits were used to locate the folder section in the Chicago RO, and the last two were used to locate the file drawer within that section. Once the drawer was located, the first claim folder in it was chosen to be one of the samples. This process was repeated until a total of 60 claim folders was selected.

\subsubsection{Determining Folder Characteristics}

Individual sample folders were first measured with a caliper for total thickness and content thickness. Information on the contents of each folder was then recorded. The documents contained in each folder were color coded and number coded. The color indicated the document's location in the folder (under the left, center, or right flap). The number established the document's sequential location under the folder flap. The form of each document was also described. (Folder documents that were drop filed were recorded as coming from under the center flap.) 


\subsubsection{Selecting Cullers}

The VA selected seven cullers for this study; six were from the Chicago RO, and a control culler was from the Washington, D.C., central office. The six cullers were split into two groups, each group consisting of one culler at level GS5, one at level GS9, and one at GS12. The GS5, GS9, and control cullers were responsible for culling the entire folder. The GS12 cullers were responsible for culling only the medical records located in the center of each folder. The names of the cullers in the first group were Edwards (GS5), Rutledge (GS9), and Stigler (GS12). Those in the second group were Wheatley (GS5), Phillips (GS9), and Wengerski (GS12). The name of the control culler was Simmons. Appendix A lists these groups for reference purposes.

\subsubsection{Culling Procedures}

The cullers were first given a list of instructions from the VA on what and how material should be sulled from each of the files. These instructions are provided in App. B. Each experimental group was given 30 folders to sull. The control culler was also given 30 folders, 15 from each experimental group. After each employee had culled a folder, the documents that were retained and those that were removed were noted, a number was assigned, and measurements of the folder thicknesses after culling were taken. A temporary folder with a number corresponding to the original folder number assisted in this process. The time taken for the culling process was also recorded. Once the information was documented, the folder was restored to its original state and placed with the remaining sample flles to be culled by other cullers. Once all the culling was completed, the colored and numbered labels placed on every piece of paper in the file were easily removed. The folders were restored to their original condition and returned to the Chicago RO to be refiled.

\subsection{COST-BENEFIT ANALYSIS}

The cost-benefit analysis was done to determine the benefit that each VA office could derive from culling claim folders. Data from the RPC upgrade initiative were incorporated in this analysis. The factors considered were the number of related ROs, number of filing cabinets, area occupied by existing claim folders (in square feet), annual costs per square foot, area saved by culling a certain percentage of the file contents (in square feet), number of claim folders, amount of time needed to cull (in years), cost of culling claim folders, annual benefit of culling (in dollars), number of years needed to pay back the cost of culling, annual rate of return, annual benefit of culling to the RPC (in dollars), pay-back period for culling to the RPC (in years), and annual rate of return for culling to the RPC. 


\subsubsection{Data Inputs}

The data inputs for the analysis consisted of information from the RPC upgrade initiative and the calculations derived from it. The material that was taken directly from the initiative was the list of 48 ROs and information on the number of filing cabinets, number of square feet occupied by filing cabinets, total nuinber of claim folders at each office, and annual costs per square foot.

\subsubsection{Spreadsheet Output}

The spreadsheet contains material that was taken directly from the RPC upgrade initiative and information derived from it. The derived material includes the following: area (in square feet) saved by culling a fixed percentage of the file contents (25\% to $40 \%$, in increments of $5 \%$ ), time needed to cull the claim folders (in years), cost of culling the claim folders (in dollars), annual benefit of culling (in dollars), pay-back period (in years), annual rate of return, annual benefit of culling to the RPC (in dollars), pay-back period for culling to the RPC (in years), and annual rate of return for culling to the RPC.

In calculating cost information, salaries of both the GS5- and GS9-level employees were used. Data on the different salaries were used in conjunction with data on the percentage of material that could be culled. Data on the different variables listed above were incorporated into the spreadsheet to provide the VA with benefit information.

The spreadsheet lists ROs in order, from the one that would benefit most to the one that would benefit least from culling. The annual rate of return was used as the benchmark to determine this ranking. Spreadsheets can be viewed in App. C. 


\section{ANALYSIS}

The analysis was conducted in three parts: (1) folder (or file) sample analysis; (2) statistical analysis of each culler, which covered the number of documents removed, culling time, and accuracy; and (3) cost-benefit analysis. These are described in Secs. 3.1-3.3, which follow.

The folder sample analysis was an overall analysis of the 60 claim folders that focused on the total thickness of the entire folder as well as the thickness under each flap. The mean, standard deviation, standard error, and population range for the total folder population as well as the total for each section were calculated.

The analysis of culler statistics involved similar calculations, bit they were applied to each individual culler. The results were used to compare cullers and determine if there were any significant differences among them in the amount of material they culled from each section of the folder. No statistics on the control person are provided in this section of the report. However, data on his time and accuracy and a regression analysis is provided.

The cost-benefit analysis resulted in a spreadsheet that shows which RO would benefit most from having its claim folders culled. The spreadsheet was the primary source for our recommendations.

\subsection{FOLDER SAMPLE ANALYSIS}

The mean thickness of the claim folder samples was 0.716 in.; the thinnest folder was $0.062 \mathrm{in}$. and the thickest was $5 \mathrm{in}$. The standard deviation was 0.691 , and the corresponding standard error was 0.089 in. We are $95 \%$ confident that the mean thickness of the VA claim folder population is between 0.533 and 0.892 in.

The mean thickness under the right-hand flap of the folder was $0.096 \mathrm{in}$. The standard deviation was 0.076 , and the standard error was $0.01 \mathrm{in}$. We are $95 \%$ confident that the mean thickness for the right side of the VA claim folder population is between 0.076 and $0.116 \mathrm{in}$.

The mean thickness under the center flap was 0.451 in. The standard deviation was 0.636 , and the standard error was $0.082 \mathrm{in}$. We are $95 \%$ confident that the wan thickness of the center of the VA claim folder population is between 0.283 and 0.619 in.

The mean thickness under the left-hand flap was 0.155 in. The standard deviation was 0.103 in., and the standard error was 0.013 in. We are $95 \%$ confident that the mean thickness of the left side of the VA claim folder population is between 0.128

and $0.182 \mathrm{in.}$ Appendix D provides the claim folder spreadsheet from which these statistics were calculated. 


\subsection{ANALYSIS OF CULLER STATISTICS}

The statistics on individual cullers were calculated on the basis of the total of the 30 files that each one culled. We used a $95 \%$ confidence level in calculating the confidence interval for each culler variable. The variables included the thicknesses of the right, center, and left sides of the claim folders, the time taken to cull, and the accuracy of the culler in culling the material. We also determined if any correlations existed between the number of documents culled and the amount of time that was needed to cull the claim folder and between the claim folder thickness and the percentage of material culled from the claim lolder. Appendix E reviews statistics on the percentage of material pulled and time for the six cullers. Appendix F provides the spreadsheet used to calculate culler statistics.

\subsubsection{Documents Removed and Time Required}

Edwards removed an average of $10.9 \%$ of the total thickness from each claim folder. The standard deviation for this average amount was $7.9 \%$, and the corresponding standard error was $1.46 \%$. We are $95 \%$ confident that on average, Edwards would remove $8-14 \%$ of the thickness from each claim folder. No significent difference was found in the average percentage of documents removed from each side of the claim folder. Edwards spent an average of $5.95 \mathrm{~min}$ to cull each claim folder. The standard deviation was $8.28 \mathrm{~min}$, and the corresponding standard error was $1.51 \mathrm{~min}$. We are $95 \%$ confident that on average, Edwards wou'd spend $2.86-9.04$ min reviewing each claim folder.

Rutledge removed an average of $33.1 \%$ of the total thickness from each claim folder. The standard deviation was $20.9 \%$, and the corresponding standard error was $3.8 \%$. We are $95 \%$ confident that on average, Rutledge would remove $25.2-40.9 \%$ of the thickness from each claim folder. No significant difference was found in the average percentage of documents removed from the each side of the claim folder. Futledge spent an average of $13.65 \mathrm{~min}$ to cull each claim folder. The standard deviation was $21.09 \mathrm{~min}$, and the corresponding standard error was $3.85 \mathrm{~min}$. We are $95 \%$ confident that on average, Rutledge would spend $5.77-21.53 \mathrm{~min}$ reviewing each claim folder.

Stigler removed an average of $7.2 \%$ of the total thickness from each claim folder. The standard deviation was $10.8 \%$, and the corresponding standard error was 1.97\%. We are $95 \%$ confident that on average, Stigler would remove $3.1-11.2 \%$ of the thickness of each claim folder. Stigler removed an average of $10.7 \%$ from the center of 30 claim folders. The standard deviation for this average amount was $14.2 \%$, and the corresponding standard error was 2.6\% . We are $95 \%$ confident that on average, Stigler would remove $5.4-16.0 \%$ of the center thickness of each claim folder. Stigler spent an average of $11.53 \mathrm{~min}$ on the center of ach claim folder during the culling process. The standard deviation was $26.92 \mathrm{~min}$, and the corresponding standard error was $4.92 \mathrm{~min}$. We are $95 \%$ confident that on average, Stigler would spend $1.48-21.58 \mathrm{~min}$ reviewing the center of each clain folder.

Wheatley removed an average of $27.4 \%$ of the total thickness from each claim folder. The standard deviation for this average amount was $11.1 \%$, and the corresponding standard error was $2.03 \%$. We are $15 \%$ confident that on average, Wheatley would 
remove 23.2-31.6\% of the thickness of each claim folder. We found no significant difference in the average percentage of documents removed from the each side of the claim folders. Wheatley spent an average of $6.98 \mathrm{~min}$ to cull each clalm folder. The standard deviation was $5.6 \mathrm{~min}$, and the corresponding standard error was $1.02 \mathrm{~min}$. We are $95 \%$ confident that on average, Wheatley would spend $4.90-9.06 \mathrm{~min}$ reviewing each claim folder.

Fhillips removed an average of $21.7 \%$ of the total thickness of each claim folder. The standard deviation for this was $10.4 \%$, and the corresponding standard error was $1.9 \%$. We are $95 \%$ confident that on average, Phillips would remove $17.8-25.6 \%$ of the thickness of each claim folder. We found no significant difference in the average percentage of documents removed from the each side of the claim folders. Phillips spent an average of $6.74 \mathrm{~min}$ on each clain folder during the culling process. The standard deviation was $6.25 \mathrm{~min}$, and the corresponding standard error was $1.14 \mathrm{~min}$. We are $95 \%$ confident that on average, Phillips would spend 4.41-9.07 min reviewing each claim folder.

Wengerski removed an average of $5.0 \%$ of the total thickness from each claim folder. The standard deviation was $8.8 \%$, and the corresponding standard error was $1.6 \%$. We are $95 \%$ confident that on average, Wengerskl would remove $1.7-8.3 \%$ of the thickness of each claim folder. Wengerski removed an average of $9.0 \%$ from the center of 30 claim folders. The standard deviation for this average amount was $18.9 \%$, and the corresponding standard error was $3.5 \%$. We are $95 \%$ confident that on average, Wengerski would remove 1.9-16.1\% of the center thickness of each claim folder. Wengerski spent an average of $4.58 \mathrm{~min}$ culling the center of each claim folder. The standard deviation was $5.79 \mathrm{~min}$, and the corresponding standard error was $1.06 \mathrm{~min}$. We are $95 \%$ confident that on average, Wengerski would spend 2.41-6.74 min reviewing the center of each claim folder.

The statistics identified above show that overall, there was no significant difference among cullers in the percentage they removed from under the left, center, or right flaps of the folders. However, some similarities did exist with respect to the types of documents that were culled from the files. These documents are listed in App. G.

\subsubsection{Culler Accuracy}

The chi squared values calculated suggest that there was a strong correlation between the six cullers and the control culler in what they were removing and retaining from the files. The calculation tables are given in App. H. Figures on the level of accuracy were also calculated from the contingency tables. Three types of information were calculated from each table:

- Type 1 Errors - Percentage of documents culled incorrectly: The percentage of total documents removed incorrectly by a culler with respect to the control culler, Simmons. 
- Type 2 - Percentage of documents culled incorrectly: The percentage of total documents letained incorrectly by a culler with respect to SImmons.

- Type 3 (Correct) - Percentage of documents culled accurately: The percentage of total documents removed or retained correctly by a culler with respect to Simmons.

The last part of this evaluation was conducted to identify the documents that Simmons had removed and the other cullers had retained and those that Simmons had retained and the other cullers had removed. We are looking for a certain pattern of documents being removed or retained in error, which could indieate in what areas training procedures could be improved. Table 1 shows the results.

\subsubsection{Regression}

The regression analysis was completed from the spreadsheet containing all the data on folders, such as the thickness of the entire folder and the thickness under its right, center, and left flaps, and the amount of time taken to cull the entire folder and the material under its right, center, and left flaps.

The regressions for Edwards, Rutledge, and Stigler were run on Folders 1 through 30. The regressions for Wheatley, Phillips, and Wengerski were run on Folders 31 through 60. Regressions for Edwards, Rutledge, Wheatley, and Phillips were run on the total folder thickness before and after culling, total time taken for culling, and total percentage removed from the folder. Stigler and Wengerski culled only the center section of the folders. Their regressions were run on total center thickness beiore and after culling, total culling time, and total percentage removed from the folder.

TABLE 1 Culler Accuracy

\begin{tabular}{llll}
\hline & \multicolumn{3}{c}{$\begin{array}{c}\text { Percentage of } \\
\text { Documents }\end{array}$} \\
& $\begin{array}{l}\text { Totaled } \\
\text { Type 1 }\end{array}$ & $\begin{array}{c}\text { Type 2 } \\
\text { Errors } \\
\text { Culler }\end{array}$ & $\begin{array}{l}\text { Type 3 } \\
\text { Errors } \\
\text { Eorect }\end{array}$ \\
\hline Edwards & 0.5 & 34.9 & 64.6 \\
Rutledge & 2.3 & 13.4 & 84.3 \\
Stigler & 1.0 & 38.7 & 60.3 \\
Wheatley & 4.6 & 25.6 & 69.8 \\
Phillips & 0.9 & 32.3 & 66.8 \\
Wengerski & 0.9 & 41.1 & 58.0 \\
\hline
\end{tabular}


The results for the regression analysis revealed a significant relationship between total folder thickness and total culling ticne. No significant relationship existed between total folder thickness and the total percentage removed from a folder. The regression analysis table is in App. I.

\subsection{COST-BENEFIT ANALYSIS}

The cost-benefit analysis was corducted by changing two variables in the costbenefit spreadsheet to identify the ROs that would benefit most from culling their claim folders. These variables were the GS-level salary and the amount of space (square feet) that would be saved at an RO by culling a specific pereentage of the claim folders. All other variables in the spreadsheet were dependent upon the above-named value chariges.

The GS-level salary variable was changed from the GS5 to GS9 step 5 salary. The square feet saved was determined by culling a certain percentage of the claim folder in increments of $5 \% ; 25 \%$ was the minirum percent culled and $40 \%$ was the maximum percent culled. The GSj and GS9 salaries were used with all percentage increments to determine culling costs. 


\section{CONCLUSIONS}

Our findings from this study are as follows:

- A significant correlation exists between the size of a folder and the amount of time it takes to cull it. Trained cullers took an average of eight minutes to cull each claim folder.

- No correlation exists between the size of a folder and the percentage of material removed. There is also no significant correlation between the percentage of material removed from a folder and its location in the folder. These fir dirgs suggest that the VA should treat all its claim folders as one continuous folder.

- The GS5- and GS9-level cullers were very accurate. When errors did occur, cullers were more likely to retain documents that si:ould have been removed than remove documents that should have been retained.

- There was no significant advantage in having a GS12-level employee cull only the center of the claim folders to remove insignificant medical documents over having a GS5- or GS9-level employee remove duplicate medical documents.

- Between $25 \%$ and $40 \%$ of the material in a claim folder can be culled.

In the most beneficial scenario, a GS5-level employee would remove $40 \%$ of the documents in a folder. The Boston RO would obtain the greatest benefit under this scenario. At Boston, it would cost $\$ 193,000$ or more to cull all the claim folders, take 10.8 person-years, result in a payback period of 1.5 years, reap an annual benefit of $\$ 125,000$ or more (this can be considered the annual cost of not culling), and result in an annual rate of return of $65 \%$.

In the least beneficial scenario, a GS9-level employee would remove only $25 \%$ of the documents. Once again, the Boston RO would obtain the greatest benefit under this scenario. It would cost $\$ 290,000$ or more to cull all the claim folders, take 10.8 personyears, result in a payback period of 3.7 years, reap an annual benefit of $\$ 78,000$ or more, and result in an annual rate of return of $27 \%$.

If the removed documents were to be transferred to the St. Louis RPC for storage, the best scenario would be a GS5-level employee removing $40 \%$ of the documents in the claim folder. Under this scenario, the payback period for culling to the RPC from Boston would be 1.8 years, the annual rate of return would be $55.7 \%$, and the yearly benefit would be $\$ 107,000$ or more. The least beneficial scenario would be a GS9level employee removing only $25 \%$ of the documents. Under this scenario, the payback period for culling to the RPC from Boston would be 4.3 years, the annual rate of return would be $23.2 \%$, and the annual benefit would be $\$ 67,000$ or more. 
The benefits from culling at each $\mathrm{RO}$ are presented in the cost-benefit analysis spreadsheet of App. C. This spreadsheet can be used by VACO to help it determine the culling plans to be used at various ROs in the future. 
APPENDIX A:

CULLERS' NAMES AND GOVERNMENT SERVICE LEVELS

\begin{tabular}{clc}
\hline Group & Name & $\begin{array}{c}\text { Government } \\
\text { Service } \\
\text { Level (GS) }\end{array}$ \\
\hline 1 & Edwards & 5 \\
& Rutledge & 9 \\
& Stigler & 12 \\
2 & Wheatley & 5 \\
& Phillips & 9 \\
& Wengerski & 12 \\
\hline
\end{tabular}


APPENDIX B:

INSTRUCTIONS TO CULLERS*

* Retyped verbatim from VBA instructions for purging material from folders. 


\section{APNENDIX B: \\ INSTRUCTIONS TO CULLERS}

\section{B.1 OVERVIEW}

VBA policy provides that we will flle and retain only essential material in our claims related folders. Material of nonrecord value is not part of our official records. The intent of this project is to provide data for a cost analysis projection. Your purpose is to remove all material from a folder which is redundant or of nunrecord value. Disposal of removed material is not part of this test. Removed material will be disposed of later, in accordance with Records Control Schedule VB-1, Part I, item 13-052. A copy of item 13-052 is attached for review purposes only.

\section{B.2 INSTRUCTIONS}

Remove all material that serves no useful purpose, or has served its initial purpose and has no additional legal, administrative or historical value. If you have a doubt about removing something and cannot resolve it within 30 seconds, leave the document in the folder. The following instructions are intended as guidelines for conducting the folder purging test project.

1. Remove duplicate coples of documents in the folder unless they contain notations of record value. Only one copy of a certlfied document is needed. Remove all other copies, certified or not.

2. Remove worksheets, control or suspense coples of VA forms, form letters, diary slips, routing slips, and letters of transmittal which have served their purpose and have no record value. Some examples:

- Diary forms and suspense copies of correspondence

- Reference and routing slips including transfer slips - VAF 707216a).

- Control records such as folder flashes that are no longer used.

- Incoming transmittal or cover letters with no evidentiary value.

- Envelopes and returned original letters with no evidentiary value.

- Requests for clvil service preference certificates. 
- VA forms and letters requesting evidence (ret'd originals or file coples)

- Target prints not specifically required by current direotives.

- Forms or correspondence containing change of address information only.

- Green control copies of VA 21-2507 and rating board worksheets.

- Duplicates SMRs (1.e. periodic exams, discharge exams, etc.).

- Duplicate private medical reports (i.e. med. hist., hospitalization, etc.). 
$21 / 22$

APPENDIX C:

COST-BENEFIT SPREADSHEETS 
TABLE C.1 CBA 25\% GS5

\begin{tabular}{|c|c|c|}
\hline AEOIOIIAL OPPICE & $\begin{array}{l}\text { OF PILE } \\
\text { CABIHIETS }\end{array}$ & $\begin{array}{l}\text { SQ. PEET } \\
\text { OCCUPIEDO }\end{array}$ \\
\hline BOATCH & 1395 & $948 \%$ \\
\hline WASHIHOTOII & 1800 & 11200 \\
\hline LOS ARAELES & 2581 & 17048 \\
\hline PROVIDEHOE & 308 & 2978 \\
\hline SAII PRALICISOO & 2100 & 11700 \\
\hline HEW YORK & 2358 & 10808 \\
\hline PITTSBURAH & 800 & 0302 \\
\hline SAH QIEOO & 713 & 991 \\
\hline SEATTLE & 1300 & 9100 \\
\hline MAHICHESTER & 220 & 1982 \\
\hline IHOIANAPOLIS & 1060 & 7420 \\
\hline CLEVELAIID & 2348 & 10438 \\
\hline ST. LOUIS & 1442 & $1009 \mathrm{~A}$ \\
\hline HOUSTOII & 1795 & 12495 \\
\hline HOIOLULUJ & 198 & 1326 \\
\hline DETROIT & 1839 & 11445 \\
\hline SUPFALO & 958 & 0592 \\
\hline HARTFORD & 395 & 0.765 \\
\hline WIIISTOU SALEII & 1432 & 10024 \\
\hline AL EUGUERCUE & 480 & 3279 \\
\hline AIICHORAQE & 78 & 546 \\
\hline DES HOLHES & 923 & 3831 \\
\hline BALTIMORE & 530 & 3920 \\
\hline$J A C \times 3011$ & 790 & 3530 \\
\hline PDRTLAIIO & BBO & 4620 \\
\hline ST, PETERSGURQ & 3430 & 20010 \\
\hline HOHTOOHERY & 1340 & 9380 \\
\hline IIEHARK & 1104 & . 7728 \\
\hline IIASHVILLE & 1199 & 8.393 \\
\hline MEH OALEAHIS & 978 & 6832 \\
\hline SALT LAKE CITY & 324 & 2129 \\
\hline 3T, PAUL & 747 & 5229 \\
\hline DEIIVER & 805 & 3639 \\
\hline FAROO & 214 & 1499 \\
\hline ROALIOKE & 1088 & 7818 \\
\hline REHLO & 255 & 1785 \\
\hline LOUISVILLE & 987 & 8909 \\
\hline CMICAOO & 1797 & 12579 \\
\hline HUSKOOEE & 979 & 6893 \\
\hline ATLAIITA & 1476 & 10332 \\
\hline WICHITA & 580 & 1080 \\
\hline DOLUPIEIA & 840 & 5936 \\
\hline HUHITIMATOH & 518 & 3833 \\
\hline LIIICOLH & 387 & 2709 \\
\hline Barge & 200 & $144 ?$ \\
\hline WACO & 2094 & 14378 \\
\hline PHILAOELPHIA & 1890 & 11872 \\
\hline ST, LOUIS RPC & 45517 & 318819 \\
\hline
\end{tabular}

AIIHUAL COST

PEA SQ. FT.
So. FT, SAVED NUHEEA OF

BY OULLIIIC O FOLOENS

$$
\begin{array}{r}
39.2 \\
19.93 \\
25.3 \\
20.49 \\
24.28 \\
21.87 \\
30.87 \\
19.93 \\
21.54 \\
18.2
\end{array}
$$

14.42

17.8

12.59

13,34

20.72

14.93

14.44

20.57

18.18

14,04

13.48

13.07

16.14

12.89

15.54

13.74

11.2

11.89

11.3

13.21

11.08

11.71

12.49

10.12

10.83

9.67

10.05

10.69

9.52

9.69

8.53

10.27

9.07

10,39

11.17

9.19

7.35

4.75
$2 \pi .0 \times$

$$
\begin{array}{r}
2370.75 \\
2800 \\
4487 \\
644 \\
3079 \\
1120.5 \\
1950.9 \\
1247.75 \\
2279 \\
395.3 \\
1858 \\
4109 \\
2523.9 \\
3123.75 \\
346.5
\end{array}
$$

2801.25

1673

691.2 :

2505

819

136.5

915,25

$$
900
$$

1382.5

115

6002.5

$23+5$

1932

2098.25

1708

532

1307.25

1400.75

374.5

$190 d$

446.25

1727.25

3144,75

1713.25

2503

1018

$148 \mathrm{~d}$

900.25

677.23

380.5

35.4. 5

2958

79054.79

143310
137927
309571
47741
291968
310224
131504

103219

203477

30399

119117

$32+250$

$14+14$

198708

33850

200558

117692

70013

203143

$89+99$

13472

69142

Q5861

99583

99316

499354

146983

138178

192297

142730

41554

101315

118530

25294

145155

30390

131237

251172

124117

204604

72079

127228

72428

62808

36401

310280

222518

10118251
PEAIOD YRE, COST OF TO CULLL

CULLIIIO

C FOLDERG

193702.0

100397.6

118574.8

6.951 .21

3817.48 .1

419497.8

164397.0

139503.7

275123.8

41102.87

101059.6

438423,5

195284

298079.6

$+\$ 789,01$

271177.0

159132.9

94885.46

274072,2

79043.15

18213,86

92135.68

$115 \mathrm{a} 3.3$

139239.9

134298.4

019748.2

190737.5

145032.2

205909.1

192987.0

30185,39

138989.2

160205.9

35538,92

196265.9

41077.19

177417.2

399012.0

167820.1

278047.6

$97+50.92$

172025.5

97929.11

$04923 .+9$

49210,28

419511.3

उ०0868. 7

21793691 
TABLE C.1 (Cont'd)

\begin{tabular}{|c|c|c|c|c|c|c|}
\hline REOIOHAL OHFTCE & $\begin{array}{l}\text { AHIUUAL BEHEFIT } \\
\text { OF CULLLIIIO }\end{array}$ & PAYBACK & ARA & $\begin{array}{l}\text { AIIIIUAL BEIIEFIT } \\
\text { CULLIIIO TO APC }\end{array}$ & $\begin{array}{l}\text { PAYBAUK } \\
\text { OULLIIIC-RPO }\end{array}$ & $\begin{array}{l}\text { AAR } x \\
\text { CULLIIIO-RPO }\end{array}$ \\
\hline BOsTOII & 78708,9 & 2.102009 & 40,01722 & 67447.83 & 2.873065 & 34.80602 \\
\hline WASHIIIOTOH & 55880 & 3.33615 .5 & 29.97182 & 12580 & 1.370703 & 22.83780 \\
\hline LOS AIIQELES & 194418,9 & 3.058279 & 27.33535 & 93108.25 & 4.499717 & 22.24339 \\
\hline PROVIDENOE & 17059.56 & 3.783873 & 26.42794 & 14000,98 & 1.010016 & 21.08907 \\
\hline SAII FRAIICISCO & 89229 & 4.272090 & 23,40442 & 71772.75 & 3.311803 & 18.82571 \\
\hline NEW YORK & 90246.55 & 4.847909 & 21.31504 & 70045.68 & $3.93 \% 180$ & 10,81214 \\
\hline PITTSBURCH & 32048.83 & 5.126148 & 19.30782 & 24683,98 & 8.053421 & 19.02489 \\
\hline SAll OLECO & 24892.61 & 3.800832 & 17.83801 & 18985.8 & 7,398704 & 13.58934 \\
\hline SEATTLE & 49003,3 & $3.01 \times 374$ & $17.811+3$ & 30197.25 & $7,205.113$ & 19.98385 \\
\hline MAIICHESTER & 7198.1 & 5.710239 & 17.51240 & 3319.475 & 7.726006 & $12,9+185$ \\
\hline ZIIO IAHIAPOLISS & 06749.1 & 0.021122 & 16,80019 & 17997.85 & 8.978757 & 11.13739 \\
\hline CLEVELAIIO & 72318.4 & 0.002392 & 16.49513 & 52800.65 & 0.303354 & 12.04332 \\
\hline ST, LOUIS & 31922.27 & 0.116857 & 16.34828 & 10935.55 & 9.794714 & 10,20958 \\
\hline HOUSTOII & 42907.95 & 0.305782 & 15.95991 & 27770.13 & 9.874982 & 10,33993 \\
\hline HCHOLULU & 7179.48 & 6.374976 & 19.68833 & 5533.005 & 0.271102 & 12.09028 \\
\hline DETAOIT & 11973,98 & 0.922780 & 13.33093 & 27983.02 & 9.890788 & 10.31909 \\
\hline BUFFALO & 24174.85 & 0.582578 & 19.19181 & 16229,1 & 9.008009 & 10.19783 \\
\hline HARTFORD & 14219.01 & 0.057688 & 15.02027 & 10935,37 & 0.056851 & 11,53181 \\
\hline WIIISTON SALEM & 40496,98 & 0.782539 & $14.7+374$ & 28593.48 & 9.500120 & 10.41002 \\
\hline AL SUOUERGUE & $11+98.76$ & 6.974099 & 14.34744 & 7608,31 & 10.38978 & 9.829767 \\
\hline AIICHORAOE & 2318,425 & 7.232957 & 13,32980 & 1870.03 & 9.740735 & 10.29616 \\
\hline OES NOIHES & 12094.91 & 7.257909 & 13.77807 & 8347.08 & 11.03807 & 9.059553 \\
\hline GALTEHORE & 15817.2 & 7.322818 & 13.55831 & 11102.2 & 10.37638 & 9.837284 \\
\hline JACKSOII & 17488.62 & 7.399888 & 13.33250 & 10921.75 & 11.93317 & $0.43 c 810$ \\
\hline PCRTLLAHO & 17948.7 & 7.181679 & 13,38598 & 12482.13 & 10.77528 & $9,280 d 98$ \\
\hline ST, PgTERSBURa & 82474.35 & 7.514412 & 13,30775 & 53382,47 & 14.40 .178 & $0,70718 \mathrm{~A}$ \\
\hline MOHTGCHERY & 26254 & 7.586919 & $13.213 d 1$ & 15125,25 & 13.13945 & 7.810064 \\
\hline MEYIARK & 22974.48 & 8.133225 & 12.25524 & 13794.49 & 13.54398 & 7,383391 \\
\hline MASHVILLE & 24129.87 & 8.933371 & 1.1 .71869 & 11183.19 & 14.93833 & 0.978368 \\
\hline MËYI ORLEAIIS & 22562.88 & 0.553374 & 11.69139 & 14449,68 & 13,35580 & 7.407383 \\
\hline DALT LAKE OITY & 6920,16 & 0.939913 & 11.24970 & 3793.18 & 14.81237 & 6.731114 \\
\hline ST, PAUL & 15307.89 & 0.948929 & 11.17492 & 9098,40 & 19.09631 & 0.641730 \\
\hline DEIIVEA & 17505.29 & 9.108458 & 10.97880 & 1090.3 .72 & 11.89027 & 0.903520 \\
\hline FAFOO & 3799.94 & 9.377174 & 10.38419 & 2011.065 & 17.87169 & 5,698788 \\
\hline ROAHIOKE & 20820,32 & 9.918082 & 10.50631 & 11578.32 & 16.93408 & 3.098203 \\
\hline AE110 & 4319.237 & 9.319101 & 10.30510 & 2195.95 & 18,70929 & 0.344938 \\
\hline LOUISVILLE & 18395.21 & 9.040390 & 10.36858 & 10190.77 & 17.41253 & 5.742989 \\
\hline CHIEACOO & 33617,37 & 10.10230 & 9,898735 & 18879.91 & 18.18073 & 3.300320 \\
\hline HUS KOOEE & 16210.14 & 10.29931 & 9.719919 & 0172.202 & 20.53548 & 4.969518 \\
\hline ATLAIITA & 23029.27 & 11.05296 & 9.047345 & 12780.02 & 21.98091 & 4.812372 \\
\hline WICHITA & 0057.95 & 11.25858 & 0.893090 & 3830.7 & 29.40175 & 3,938735 \\
\hline COLLITEIA & 15240.68 & 11.28733 & 8.859490 & 8191.88 & 21,00018 & 4.781968 \\
\hline HUHTIMOTOH & 8237.827 & 11.88701 & 0.412117 & 3923.04 & 24.99848 & 1.008093 \\
\hline LIIICOLII & 7038,627 & 12.08877 & a. 285043 & 3819.89 & 22.23308 & 4.497801 \\
\hline 80198 & 4028.785 & 12.22271 & $8.191+88$ & 2314.41 & 21.26000 & 4.702340 \\
\hline WACO & 33033,15 & 12.09989 & 7.974208 & 15959,98 & 28.28508 & 3.004295 \\
\hline PHILADELPHIA & 21814.0 & 13.79186 & 7,250092 & 7716.9 & 38.98953 & 2.384856 \\
\hline ST, LCUIS RPC & 378360.0 & 57.80040 & 1.738099 & 0 & ERR & 0 \\
\hline
\end{tabular}


TABLE C.2 CBA 25\% GS9

\begin{tabular}{|c|c|c|c|c|c|c|c|}
\hline REOIOIIAL OPFIOE & $\begin{array}{l}\text { OP PILE } \\
\text { CABIHETS }\end{array}$ & $\begin{array}{l}\text { SQ, FEET } \\
\text { OCOUPIEO }\end{array}$ & $\begin{array}{l}\text { AIIHUAL COST } \\
\text { PEA SO, FT, }\end{array}$ & $\begin{array}{l}\text { Sa. FT, SAVED } \\
\text { QY CULLIIIO } \\
\text { as.0 } \mathrm{x}\end{array}$ & $\begin{array}{l}\text { HUMEER OF } \\
\text { C POLDERS }\end{array}$ & $\begin{array}{l}\text { PEAIOO YAS, } \\
\text { TO CULLL }\end{array}$ & $\begin{array}{l}\text { COST OF } \\
\text { CULLIIIO } \\
\text { O FOLDERS }\end{array}$ \\
\hline BOsTOII & 1345 & g. & 33,2 & 2370,75 & 143318 & 10.78587 & 290873.1 \\
\hline HASHLHOTOH & 1800 & 11200 & 19.95 & 2800 & 137827 & 10.35320 & 279538.4 \\
\hline LOS AllOELES & 2504 & 17.948 & 25,5 & 1487 & 309871 & 23,25411 & 627862,3 \\
\hline PROVIDEIICE & 968 & 2578 & $28,4 y$ & 014 & $177+1$ & 3.506178 & 98826.91 \\
\hline SAII FMAIICISO0 & 2100 & 14700 & 24.28 & 3875 & 281905 & 31,18040 & 571072.6 \\
\hline IEEW YORK & 2358 & 10500 & 21.87 & 4125,5 & 310224 & 23.30321 & 029185.7 \\
\hline PITTISBUROH & 808 & 8202 & 20.87 & 1530.3 & 121304 & 9.127061 & 248430.5 \\
\hline SAll OIHOO & 713 & 4991 & 19.98 & 1247.73 & 103219 & 7,753539 & 209345.5 \\
\hline SEATTLE & 1300 & 9100 & 21.51 & 2275 & 203477 & $15.39+85$ & 112999.7 \\
\hline MAIICHESTER & $22 \theta$ & 1982 & 18.2 & 395.3 & 30399 & $2.293+92$ & 01894,30 \\
\hline INOI AIIAPOLIS & 1080 & 7420 & 14,42 & 1095 & 119117 & $0.9+7753$ & $2 \$ 1589,4$ \\
\hline CLEVELAIID & $23+\theta$ & 10430 & 17.3 & 4109 & 324250 & $24,356 \pm 2$ & 637833.8 \\
\hline ST. LOUYB & 1442 & 10094 & $12 . a:$ & 2923.5 & $14+414$ & $10.8+2$ & 292999 \\
\hline HOUSTOH & 1705 & 12495 & 13.61 & 3123,75 & 193708 & $14,925+2$ & 403013.4 \\
\hline HOHOLUL'J & 198 & 1356 & $20,7=$ & 348,5 & 33850 & 2.342723 & $\cos 33,52$ \\
\hline OETROIT & 1835 & $11 d d s$ & 14,33 & 2981.29 & 200998 & 19.06539 & 408758.9 \\
\hline BUFPALO & 938 & 0022 & 14,49 & 1673 & 117092 & 0.810713 & 239998.2 \\
\hline HAEKTFORD & 395 & 2703 & 20.97 & 691,25 & 70013 & 9.219192 & 141998.1 \\
\hline WIIISTOII SALEH & 1432 & 10024 & 18.18 & 2506 & 203143 & 18.25999 & 412008.3 \\
\hline AL QUQUERQUE & 469 & 3278 & 14.21 & 819 & $98+59$ & 4.391285 & 118584.7 \\
\hline AIICHORAOE & 78 & 548 & 18,13 & 130.3 & $13+72$ & 1.011991 & 27323.49 \\
\hline DIS NOYIIES & 323 & 3561 & 13.97 & 915,25 & 09142 & 5.119547 & 139203.2 \\
\hline BALTIHORE & 580 & 3920 & 10.14 & 900 & 85861 & 0.434619 & 173704.9 \\
\hline JACKSOII & 790 & 5530 & 12.93 & 1302.3 & 95583 & 7.179943 & 193858.4 \\
\hline PURTLAIID & 680 & 1620 & 13,94 & 1155 & 99316 & $7,1803 \pm 3$ & 201429.5 \\
\hline ST, PETERSBURO & 3430 & 24010 & 13,74 & 6002.9 & 193354 & $34,+3034$ & 929819.3 \\
\hline MOHTOOHERY & $13+0$ & 9380 & 11.2 & 2345 & 140283 & 11.04097 & 298109.3 \\
\hline HEYAARK & 1101 & 7728 & 11,89 & 1932 & 139178 & 10.37946 & 290249.3 \\
\hline MASHVILLE & 1199 & 8393 & 11.3 & 2098.25 & 152297 & 11.03939 & 308863.7 \\
\hline HEW ORLEAHIS & 976 & 8032 & 13.21 & 1708 & $1+2730$ & 10.72190 & 289480,5 \\
\hline SALT LAKE CITY & 304 & 2129 & 11.98 & 392 & 41354 & 3.121427 & 04278.53 \\
\hline ST. PAUL & 747 & 3229 & 11.71 & 1307,25 & 101315 & 7.010518 & 205483.9 \\
\hline OE!IVE? & $80:$ & 3539 & 12.49 & 1408,75 & 118930 & 8.903931 & 240398.3 \\
\hline PAROO & 214 & 1498 & 10.12 & 374.9 & 25284 & 1.974384 & 53308,39 \\
\hline ROA!ICKE & 1080 & 7018 & 10,83 & 1904 & 145155 & 10.90368 & 294348,8 \\
\hline REIIO & 259 & 1785 & 9.87 & $4+5,25$ & 30380 & 2.242055 & 01615.77 \\
\hline LOUISVILLE & 987 & 8909 & 10.63 & 1727.29 & 131237 & 9.858178 & 266170.8 \\
\hline CHICAOO & 1797 & 12579 & 10.69 & 3144.75 & 291172 & 18.38739 & 309419.2 \\
\hline MUSKOGEE & 979 & 8893 & 9.12 & 1713.25 & 124117 & 9.323 .342 & 251730.2 \\
\hline ATLAIITA & 1470 & 10332 & 9.99 & 2583 & 201504 & 19.36931 & 414971.4 \\
\hline WICHITA & 580 & 1080 & $8,: 3$ & 1015 & 72274 & 5.414324 & 140188.3 \\
\hline COLUPIBIA & 848 & 5936 & 10.27 & 9484 & 127229 & 9.397032 & 290039.8 \\
\hline HUHTIIIATOH & 519 & 3833 & 9.07 & 908.25 & 72420 & 9.440490 & 148992.1 \\
\hline LIIICOLII & 387 & 2709 & 10.39 & 677.25 & 02900 & 4.717971 & 127385.2 \\
\hline Bor3a & 200 & 1442 & 11.17 & 300.5 & 38401 & 2.734347 & 73827.39 \\
\hline WACO & 2054 & 14378 & 9,19 & $359+.9$ & 310260 & 23.30635 & 029271.8 \\
\hline PHZLADEI.PHIA & 1090 & 11872 & 7.39 & 2969 & 222518 & $10,71+81$ & 451300.0 \\
\hline ST, LOUIS RPC & 19317 & 313819 & 1.7! & 79054,75 & 16113251 & 1210.760 & 32590537 \\
\hline
\end{tabular}


TABLE C.2 (Cont'd)

\begin{tabular}{|c|c|c|c|c|c|c|}
\hline REQIOIIAL OFFICE & $\begin{array}{l}\text { AHIIUAL BEHEFIT } \\
\text { OF. CULLLING }\end{array}$ & PAYAACK & ARR & $\begin{array}{l}\text { AIIIUUAL BEHIEFIT } \\
\text { CULLIHO TO RPO }\end{array}$ & $\begin{array}{l}\text { PAYBAOK } \\
\text { GULLIHG-APC }\end{array}$ & $\begin{array}{l}\text { ARR : } \\
\text { CULLIIIO-APC }\end{array}$ \\
\hline BOSTOH & 78700.9 & $3.89301 d$ & 27.07814 & 87447.83 & 4,309598 & 23.20401 \\
\hline WASHIIIOTON & 55800 & 5.004232 & 19,98308 & 42300 & 0.560035 & 15.22920 \\
\hline L.OS AllaELES & 114418.5 & $3: 187419$ & 18.22390 & 03105.29 & 0.743979 & 14.92992 \\
\hline PROVIDEIICE & 17059,58 & 5.875809 & 17.81863 & 14000.38 & 0.915924 & 14.45938 \\
\hline SAN PRAIICISCO & 89229 & 0.409044 & $15.6029 d$ & 71772.79 & 7.987824 & 12.53047 \\
\hline NEW YORK & 90246,33 & 0.971983 & 14.34330 & 70845.08 & 8.900230 & 11.22809 \\
\hline PITTSBURAH & 32048,89 & 7.689223 & 13.00921 & 24683.96 & 9.993432 & 10.01859 \\
\hline SAII DIEOO , & 24892.01 & 8. 409048 & 11.89007 & 18985.8 & 11.03809 & 9.059505 \\
\hline SEATTLE & 49003.5 & $8,421 \leq 98$ & $11.97+29$ & 38197.28 & 10.80408 & 9.255771 \\
\hline MAIICHEBTER & 7199.1 & 0.505353 & $11.67+93$ & 5319,475 & 11.59029 & Q. $42790 d$ \\
\hline IHOLAMAPOLIS & 26749.4 & 9.031693 & 11.07213 & 17937.95 & 13,46813 & 7.424932 \\
\hline OLEVILAIIO & 72318.4 & 9.093539 & 10.99679 & 82800.65 & 12.45903 & 0.229833 \\
\hline 31, L2UIS & 31922.27 & 9,175235 & 10.89884 & 19935.85 & 14.69207 & 0.903392 \\
\hline HOUSTOH & 42507.99 & $9.49364:$ & 10,57234 & 27770.13 & 14.91247 & 0.990823 \\
\hline HOHOLUL'J & 7179.48 & 9.982404 & 10.49753 & 3833.005 & 12.40865 & 0.280191 \\
\hline OETPOOIT & 11573.96 & 9,784141 & 10.22092 & 27983.02 & 14.53815 & 6.979399 \\
\hline BUFFALO & 24174.85 & 9.873997 & $10.127 i t$ & 18228.1 & $1+.70900$ & $0.7395 \pm 1$ \\
\hline HAHTFORO & 14213.01 & 9.990502 & 10.01351 & 10935.57 & 12.99497 & 7.701200 \\
\hline WIIISTCII SALEN & 10496.98 & 10.17390 & 9.829160 & 28593.40 & 14.40918 & 0.940019 \\
\hline ALSUQUEFQUE & 11.99 .76 & 10.31109 & 9.699297 & 7608.51 & 15.58397 & $6.11717 \mathrm{~g}$ \\
\hline AILCHORAQE & 2519.425 & $10.849+3$ & 9.217009 & 1870.08 & 14.61110 & $6.9+1110$ \\
\hline DES MOYIIES & 12594.51 & 10.82998 & 9.135391 & 8347.08 & 18.55710 & 6.039702 \\
\hline BALTIHORE & 15817.2 & 10.98392 & 9.104211 & 11182.2 & $19,56 \mathrm{~A} 58$ & $6.42+a+2$ \\
\hline JACKSOII & 17488.82 & $11.09 \pm 93$ & 9.221338 & 10921.75 & 17.74976 & 5.533877 \\
\hline PORTLAIIO & 17948.7 & 11.22281 & 8.910055 & 12482.45 & 10.16292 & 6.198999 \\
\hline ST. PETERSGURG & 82474.35 & 11.27151 & 8.871840 & 53902.47 & 17.227 .14 & 5.904792 \\
\hline MOITOOOHERY & 28284 & 11.35037 & 0.910273 & 15125.25 & 19.70918 & 5.073776 \\
\hline HEWARK & 22971.48 & 12.19933 & 0.198830 & 13794.48 & 20.31597 & 4. 922234 \\
\hline MASHVILLEE & 24129.87 & 12.80005 & 7.912465 & 14163.18 & 21.80730 & 4.535577 \\
\hline NEIN ORLEAIIS & 22562.89 & $12.83 \mathrm{COB}$ & 7.794195 & 14449.88 & 20.03370 & 1.991549 \\
\hline SALT LAYE GITY & 0320.16 & $13.33+97$ & 7.499133 & 3793.10 & 22.21855 & 4.500742 \\
\hline ST. PAUL & 15307.89 & 13.42339 & 7.419830 & 9098.48 & 22.98447 & 1.127820 \\
\hline DEHVE? & 17595.28 & 13.55298 & 7.319298 & 10903.72 & $22.0 . \cdot 40$ & 4.538580 \\
\hline FARQOO & 3789.94 & 14.09570 & 7.109481 & 2011.085 & 29.50754 & 3.772910 \\
\hline ROAHOKLE & 20020.32 & 14.27712 & 7.004211 & 11578.32 & 25.43112 & 3.932189 \\
\hline REHIO & 4315.237 & 14.27899 & 7.003482 & 2195.55 & 29.08393 & 3.963292 \\
\hline LOUIEVILLE & 10395.21 & 14.48957 & 0.911055 & 10190.77 & 26.11880 & 3.928599 \\
\hline CHICAOO & 33817,37 & 15.15345 & 6.599157 & 18679.81 & 27.27110 & 3.358984 \\
\hline MUSKOOEE & 18310.14 & 45,43397 & 6.479213 & 0172.202 & 30.8032 .3 & $3.248+12$ \\
\hline ATLAIITA & 25029.27 & 15.57344 & 0.031563 & 12760.02 & 32,52122 & $3.07+914$ \\
\hline WICHITA & 8657.95 & 16.88437 & 3.922460 & 3836.7 & 38.10283 & 2.324490 \\
\hline COLUIIBIA & 15240.68 & 18.93099 & 3.900327 & 8191.68 & 31,50024 & 3.174578 \\
\hline HUHTINOTOH & 8237.027 & 17.83142 & 5.808078 & 3923.64 & 37.43772 & 2.671102 \\
\hline LIIlCOLH & 7036.627 & 18.10319 & 5.323895 & 3619.69 & 33.34982 & 2.992534 \\
\hline BOIsE & 1026.785 & $18.33+07$ & 5.454324 & 2314.41 & 31.89900 & $3.13+893$ \\
\hline WACO & 33033.45 & 19.04993 & 5.249472 & 15959.58 & 39,42910 & 2.939197 \\
\hline PHILACELPHIA & 21814.8 & 20.68779 & 4.073758 & 7716.8 & 38.48279 & 1.709904 \\
\hline ST, LOUIB RPC & 378380.0 & 88.40000 & 1.197399 & 0 & ERP & 0 \\
\hline
\end{tabular}


TABLE C.3 CBA 30\% GS5

RESIOIAL OPFICE

\begin{tabular}{|c|c|c|}
\hline BOSTON & 1355 & 9483 \\
\hline HASHIHATOH & 1600 & 11200 \\
\hline LOS AIIOELES & 2584 & 17948 \\
\hline PROVIDEIICE & 368 & 2378 \\
\hline SAII FRAIICISOO & 2100 & 14700 \\
\hline NEH YOAK & 2358 & $18 \leq 08$ \\
\hline PITTSBUROH & 886 & 6202 \\
\hline SAII DIEQO & .713 & 4991 \\
\hline SEATTLE & 1300 & $910 \%$ \\
\hline MAIICHESTER & 220 & 1992 \\
\hline IHOIAHAPOLIS & 1080 & 7420 \\
\hline CLEVELAHIO & 2348 & 15436 \\
\hline ST, LOUIS & $14 d 2$ & 10074 \\
\hline HOUSTOHI & 17 as & 12495 \\
\hline HOHOLULU & 198 & 1338 \\
\hline DET'ROIT & 1635 & $11+\$ 5$ \\
\hline SUFFALO & 956 & 6692 \\
\hline HARTFORD & 395 & 2789 \\
\hline WIIISTOH SALEH & $1+32$ & 10024 \\
\hline ALBUQUERGUE & 468 & $3 \approx 78$ \\
\hline AIICHORAOE & 78 & 545 \\
\hline DES MOIHES & 523 & $3 E 81$ \\
\hline BALTIIOORE & 560 & 3920 \\
\hline JACKSOHI & 790 & 5530 \\
\hline PONTLAIIO & 660 & 4820 \\
\hline ST, PETERSBURO & 3430 & 24010 \\
\hline MOIITAOHERY & 1340 & 9390 \\
\hline MEIYARK & 1104 & 7729 \\
\hline HASHVILLE & 1199 & 8393 \\
\hline MEH CRLEAHS & 976 & 6332 \\
\hline SALT LAKË CITY & 304 & 2128 \\
\hline ST. PAUL & 747 & 3229 \\
\hline DEIIVER & 805 & 5635 \\
\hline FARCO & 214 & 1498 \\
\hline ROAIIOKE & 1088 & 7816 \\
\hline REHY & 255 & 1785 \\
\hline LOUISVILLEE & 987 & 8229 \\
\hline CHICAOO & 1797 & 12579 \\
\hline MUSKCOEE & 979 & 6353 \\
\hline ATLAIITA & 1475 & 10332 \\
\hline WICHITA & 580 & 4080 \\
\hline COLLIABIA & 849 & 5935 \\
\hline HUITEHLTOH & 519 & 3833 \\
\hline LIIICOLII & 387 & 2709 \\
\hline BOISE & 208 & 1442 \\
\hline WACO & 2054 & 14373 \\
\hline PHILADELPHIA & $1890^{\circ}$ & 11872 \\
\hline ST, LOUIS RPC & 45517 & 318819 \\
\hline
\end{tabular}

AlHIUAL COST PER SQ, FT.
S2. FT, SAVEO IUUIEER OF BY CULIIIIIO 30.0 \%

\begin{tabular}{|c|c|c|}
\hline 33.2 & 2844.9 & 143318 \\
\hline 19,98 & 3380 & 107927 \\
\hline 25.5 & 3384,4 & $3095 \% 1$ \\
\hline 2.8 .49 & 772,8 & $477+1$ \\
\hline 24.28 & 4410 & $2919 B 3$ \\
\hline 21.87 & 4951.8 & 310224 \\
\hline 20.87 & 1800.6 & $121: 0.4$ \\
\hline 19.95 & $1,197,3$ & 103219 \\
\hline 21.91 & 2730 & $203+77$ \\
\hline 19.7 & $47+6$ & 30390 \\
\hline $14,4 \%$ & 2220 & 119117 \\
\hline 17.6 & 4930.9 & $32+250$ \\
\hline $12,3:$ & 3028.2 & 104414 \\
\hline 13.94 & 3748.5 & 198708 \\
\hline 20.42 & 415.9 & 93890 \\
\hline 14.93 & 3433,5 & 200558 \\
\hline $14,4 !$ & 2007.6 & 117892 \\
\hline 20.57 & 029.5 & 70013 \\
\hline $18,1 H$ & 3007.2 & 203143 \\
\hline 14.04 & 922.8 & 32459 \\
\hline $18,+5$ & 163.8 & 13472 \\
\hline 13.87 & 1099.3 & $8 a 1+2$ \\
\hline 18.14 & 1176 & $8506 ;$ \\
\hline 12.35 & 1559 & 95583 \\
\hline 15.54 & 13.95 & 99316 \\
\hline 13.74 & 7203 & 458354 \\
\hline 11.2 & 2914 & 146983 \\
\hline 11.99 & 2319.4 & 138178 \\
\hline 11.3 & 2517.9 & 152297 \\
\hline 13.21 & 2049.6 & 142730 \\
\hline 11.89 & 838.4 & 91554 \\
\hline 11.71 & 1888.7 & 101315 \\
\hline 12.49 & 7690.5 & 118530 \\
\hline 10.12 & 449,4 & 29284 \\
\hline 10.33 & 2294.8 & 145155 \\
\hline 9.97 & 535.5 & 30380 \\
\hline 10.55 & 2072.7 & 131237 \\
\hline 10.59 & 3773.7 & 251172 \\
\hline 9.32 & 2055.9 & 124117 \\
\hline 9.59 & 3094.6 & 204604 \\
\hline 8.53 & 1218 & 72079 \\
\hline 10.27 & 1780.8 & 127228 \\
\hline 9.07 & 1089.9 & 72426 \\
\hline 10.39 & 812.7 & 62900 \\
\hline 11.17 & 432.0 & 36101 \\
\hline 2.19 & 4313.4 & 310286 \\
\hline 7.25 & 3561.5 & 222516 \\
\hline 4.75 & 95505.7 & 15112251 \\
\hline
\end{tabular}

PEALCO YRS. COST OA TO CULL CUlLLIllO C FOLOEPS

$10.76537 \quad 1937890$ $10.35332 \quad 104367,2$ 23.25d15 4185Tt.0 3.58178 645\$1.21 $21.18948 \quad 381248.4$ $23.30321 \quad 019437,0$ 9.127051; $16+287.0$ $7.753918 \quad 139503.7$ 15.28165 275143. $2.293492 \quad 41102.97^{\circ}$ 8.947755 $1610: 9.8$ 24. $49383 \quad 438451.5$ 10. 284 19525d 14, $92542 \quad 2 \leqslant 8078,8^{\circ}$ $2.442723 \quad 45783.01$ 15.05:33 274177.0 8.840713 999133.8 $5.259192, \quad 94859.46$ $15,25095 \quad 274872.2$ 4.35.129.5 $790+3.15$ $1.011821 \quad 18219.66$ $5.118647 \quad 92135.65$ $0.434523 \quad 115223.3$ $7.179943 \quad 129228.9$ $7.450355 \quad 134295.4$ $34.43034 \quad 619746.2$ $11.04097 \quad 193737.5$ $10.37935 \quad 180 \mathrm{a} 32.2$ 11.43935 205909.1 $10.72150 \quad 192987.0$ $3.121427 \quad 56185.69$ $7.510515 \quad 136999.2$ $8.303851 \quad 160255.9$ $1.97+334 \quad 35538.92$ $10.90326 \quad 198255.9$ 2.295205 41077.18 $9.853178 \quad 177447.2$ $18.35739 \quad 339512.8$ $9.323342 \quad 167920.1$ $15.36731 \quad 276647.6$ $5.41433 .1 \quad 97+58.92$ $9.557032 \quad 172025.5$ $9.440480 \quad 97928.11$ $4.717971 \quad 84923.49$ $2.73+347 \quad 49219.25$ $23.30533 \quad 413514.5$ $16.7: 421 \quad 300999.7$ $1210.750 \quad 21793091$ 
TABLE C.3 (Cont'd)

REGIOHAL OFFICE.

WASHIHGTOH

LOS AHCELES

PROVIDEHICE

SAN FRAIICISCO

NEIY YORK

PITTSEUROH

SAII DIEGO

SEATTLE

MAICHEETER

IMLIAHIAPOLIS

clevelaho

ST. LOUIS

HOUSTOH

HONOLULU

DETFE:T

BUFEALO

HARTFORO

WIIISTOH SALE!!

albliguerque

AIICHORACE

DES MOIHES

BALTIHORE

JACKSOH

PORTLAHID

ST. PETERSEURG

MOIITGOHERY

MEWARK.

MASHVILIE

MEW GRLEAHS

SALT LAKE CITY

ST. PAUL

DEHIVER

FARGO

ROAHIEK

RE:10

LOUTSVILLE

CHICAGO

MUSKOGE:

ATLAITTA

WICH:TA

COLUMEIA

MURTIHOTOII

LIIICOL!!

BOISE

WACO

PHILADELFHIA

ST. LOUIS RPC
AHIIUAL BEHEFIT PAYBACK OF CULLIHO

94450.68
67032
137302.2
20471.47
107074.8
102295.8
38458.60
29871.13
53804.2
8637.72
32099.92
80792.08
39306.73
51129.54
8515.376
49393.75
29009.32
11082.81
48595.35
13798.51
3022.11
15233.12
18990.64
20985.35
21539.44
93959.22
31516.8
27585.77
29955.85
27075.21
7584.192
18369.47
21114.34
4547.929
24744.38
5178.295
23074.25
40340.85
19572.16
30035.12
10389.54
18299.81
9395.393
8443.953
4832.142
39640.14
25177.76
454032.0

Aรก

(1)

$\begin{array}{lll}\text { AHHUAL BEIIEFIT } & \text { PAYSACK } & \text { APR: } \\ \text { CULLIHO TO RPC } & \text { CULLIHIG-RPC } & \text { CULLIHG-RFC }\end{array}$

\begin{tabular}{|c|c|}
\hline 2.0 & 48.74066 \\
\hline 2.780129 & 35.96954 \\
\hline 3.048556 & 32.80230 \\
\hline 3.153227 & 31.71353 \\
\hline 3.560580 & 29.08530 \\
\hline 3.873257 & $25.21 \leq 05$ \\
\hline 4.271790 & 23.40938 \\
\hline 4.672193 & 21.40322 \\
\hline 4.578642 & 21.27372 \\
\hline 4.758532 & 21.01489 \\
\hline 5.017602 & 19.92993 \\
\hline 5.051993 & 19.79415 \\
\hline $5.09739 i$ & 19.51791 \\
\hline $5.25+301$ & 19.02021 \\
\hline 5. 312490 & 12.92359 \\
\hline 5.435634 & 18.33711 \\
\hline 5.485481 & 18.22993 \\
\hline 5.548056 & 19.02432 \\
\hline 5.652116 & 17.69248 \\
\hline 5.728382 & 17.45693 \\
\hline 6.027464 & 16.59072 \\
\hline 6.042258 & $1 \overline{4} .5336 \bar{\varepsilon}$ \\
\hline 6.102182 & 10.38758 \\
\hline $6.1582+0$ & 16.23940 \\
\hline 6.234732 & 16.03917 \\
\hline 6.252010 & 15.95931 \\
\hline 6.305756 & 15.85850 \\
\hline 6.777637 & 14.75425 \\
\hline 7.111142 & 14.06243 \\
\hline 7.127811 & 14.02955 \\
\hline 7.408253 & 13.49844 \\
\hline 7.457441 & 13.40942 \\
\hline 7.590390 & 13.17455 \\
\hline 7.814312 & 12.79703 \\
\hline 7.931735 & 12.50759 \\
\hline 7.932534 & 12.50623 \\
\hline 8.038650 & 12.43989 \\
\hline 8.418593 & $11.378+8$ \\
\hline 8.574429 & 11.86252 \\
\hline 9.210804 & 10.35521 \\
\hline $9.320+85$ & 10.66042 \\
\hline 9.406108 & 10.63138 \\
\hline 9.906344 & 10.09454 \\
\hline 10.05731 & Q. 943011 \\
\hline 10.18559 & 9.817784 \\
\hline 10.52307 & 9.445050 \\
\hline 11.49321 & 8.750723 \\
\hline 49.00033 & 2.023318 \\
\hline
\end{tabular}

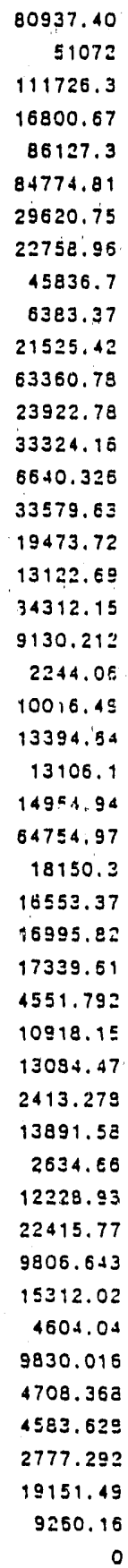

\begin{tabular}{|c|c|}
\hline 2.394221 & 41.76722 \\
\hline 3.642919 & 27.40537 \\
\hline 3.746430 & 25.62207 \\
\hline 3.842180 & 26.02588 \\
\hline 4.426569 & 22.59085 \\
\hline 4.947505 & 20.21057 \\
\hline 5.546351 & 19.02987 \\
\hline 6.132253 & 16.30721 \\
\hline 6.002250 & 18.50039 \\
\hline 6.439055 & 15.53022 \\
\hline 7.482297 & $13.36+87$ \\
\hline 6.919462 & 14.45198 \\
\hline 8.162262 & $12.251 \equiv 0$ \\
\hline $8.06248 \Xi$ & 12.40312 \\
\hline 5.892535 & 14.50234 \\
\hline 8.075640 & 12.32291 \\
\hline 8.171671 & 12.23733 \\
\hline 7.213975 & :13.26217 \\
\hline 8.005100 & 12.49203 \\
\hline $8.5 \equiv 7312$ & 11.55092 \\
\hline 8.117279 & 12.31939 \\
\hline 9.198392 & 10.97145 \\
\hline 8.645990 & 11.55471 \\
\hline 9.880979 & 10.14098 \\
\hline 0.979402 & 11.13559 \\
\hline 9.570636 & 10.44262 \\
\hline 10.94954 & 9.132797 \\
\hline $11.286 \in E$ & 8.860021 \\
\hline 12.11528 & 8.254039 \\
\hline 11.12983 & 8.984860 \\
\hline 12.34364 & 8.101336 \\
\hline 12.54693 & 7.970076 \\
\hline 12.24855 & 8.164225 \\
\hline 14.72641 & 6. $7 \cong 0519$ \\
\hline $14.1 .29+0$ & 7.077940 \\
\hline 15.59107 & 6.413925 \\
\hline 14.51044 & 6.891587 \\
\hline 19.15061 & 6.500391 \\
\hline 17.11290 & 3.243542 \\
\hline 18.05734 & $5 . \equiv 34340$ \\
\hline 21.15313 & 4.724052 \\
\hline 17.50013 & 5.714242 \\
\hline 20.79273 & 4.907934 \\
\hline 18.52757 & 5.397361 \\
\hline 17.72157 & 5.543908 \\
\hline 21.90505 & $4,5 E \leq 150$ \\
\hline 32.49042 & 3.277329 \\
\hline ESP: & \\
\hline
\end{tabular}


TABLE C.4 CBA 30\% GS9

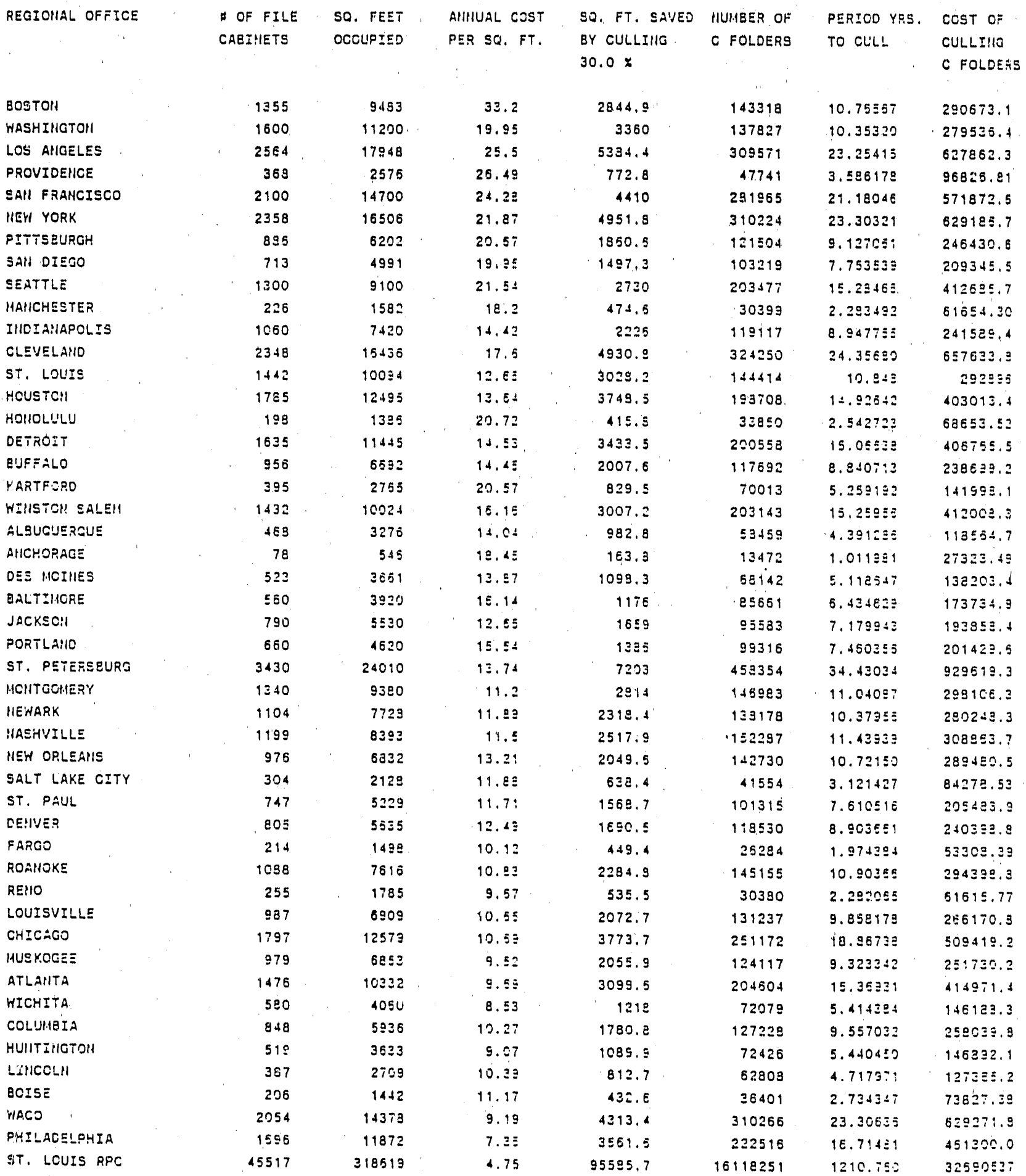


TABLE C.4 (Cont'd)

REQIOUIAL OFFICE

BOSTOH

WASHIMGTON

LOS ARGELES.

PROVIOE!ICE

SAHI FraHCISCO

HEN YORK

PITTSEURGH

SAII DIESO

SEATTLE

MAHCHESTE?

IHDIAHAPOLIS

CLEVE'LAIID

ST. LOLIS

HCUSTCI

HOHOLULU

DETROET

RUFFALC

HARTECFE

WIIISTCH SALE!

ALSUGLEPQUE

AHCHORAGE

DES MOZIIES

BALT:HCRE

JACXSCII

FOFTLAIIO

ST. PETEFSEURG

MOHTOCMERY

MEYARK.

MASHVILLE

MEIY OPIEAIIS

SALT LAXE CITY

ST. PALL

DE!IVES

FARGO

ROAIIOKE

REIO

LOUISVILLE

CHICASO

HUSKOCEE

ATLAIIT:

WICH:TA

COLUME: 2

HUHTIIGTOH

LIIlCọ!.!

ROISE

WACO

PHILACELPHIA

ST. LOUIS RPC
AIIIUAL BEIEFIT PAYBACN A ARR OF CULLIIIG

\begin{tabular}{|c|c|c|}
\hline 94450.68 & 3.077512 & 32.49377 \\
\hline 67032 & 4.170194 & $23.979 E 9$ \\
\hline 137302.2 & 4.572949 & 21.28220 \\
\hline 20471.47 & 4.729241 & 21.14235 \\
\hline 107074.8 & $5.3408>0$ & 18.72353 \\
\hline 109295.8 & 5.809896 & 17.21203 \\
\hline 39459.60 & 6. 107686 & 15.50825 \\
\hline 29371.13 & 7.003290 & 14.25281 \\
\hline 53804,2 & 7.017953 & 14.24914 \\
\hline 8537.72 & 7.137793 & $14 . \cos 92$ \\
\hline 32099.92 & 7.525403 & 13.25355 \\
\hline 86782.09 & $7.5779: 0$ & $13.1 \equiv 80$ \\
\hline 38305.73 & 7.545074 & 13.07351 \\
\hline 51129.54 & 7.es2202 & 12.53680 \\
\hline 8515.376 & 7.958720 & 12.54505 \\
\hline 49888.75 & 8.153451 & 12.25474 \\
\hline 29009.82 & 8.229222 & 12.15329 \\
\hline 17062.31 & 8.322035 & $12.21 E 21$ \\
\hline $48 \leq 95.35$ & 8.478174 & 11.79499 \\
\hline 13792.51 & 8.592573 & 11.33795 \\
\hline 3022.11 & 9.041197 & $11.050+3$ \\
\hline 15233.42 & 9.072337 & 11.02245 \\
\hline 19980.64 & 9.153273 & $10 . \$ 2505$ \\
\hline 20996.35 & Q.2373こ0 & 10. \\
\hline 21538.44 & 9.352099 & 10.59278 \\
\hline 99969.22 & 9.393015 & $10, E 4 E 20$ \\
\hline 31516.8 & 9.458549 & 10.57333 \\
\hline 27555.77 & .16553 & 9.233195 \\
\hline 29955.85 & 0.85671 & 9.374950 \\
\hline $27075 \geq 1$ & 10.69171 & 2.233034 \\
\hline 7584.192 & 11.11239 & $8, \equiv \equiv 2950$ \\
\hline 18369.47 & 19.18516 & 8. $\$ 22515$ \\
\hline 21194.34 & 11.39557 & 8.7331040 \\
\hline $45+7.928$ & 11.72140 & 8. $5: 13: 4$ \\
\hline 24744.38 & 11.89760 & 8. 425.253 \\
\hline 5178.225 & 11.89837 & e. 224154 \\
\hline 22074.25 & 12.05797 & 8. 233256 \\
\hline 4.2340 .85 & 12.82787 & $7 . \equiv: 2 \equiv 28$ \\
\hline 19572.16 & 12.85154 & 7.775055 \\
\hline 32035.12 & 13.21320 & 7.257375 \\
\hline 10399.54 & 14.07072 & 7.165952 \\
\hline 19288.81 & $14.109: 6$ & 7.087592 \\
\hline 9885.393 & 14.85951 & 6.729593 \\
\hline 8443.953 & $15.08 \leq 97$ & 6.229574 \\
\hline 4832.142 & 15.27839 & 6.543 .39 \\
\hline 39640.14 & 15.87461 & $6.259 \$ 57$ \\
\hline 25177.76 & 17.23992 & 5.250522 \\
\hline $4 \equiv 4032.0$ & 72.00050 & 1. 323279 \\
\hline
\end{tabular}

AMIIUAL BEIIEFIT PAYGACK ARP : CULLIHG TO RPO CULLIHO-RPS CULLIHG-RPC

\begin{tabular}{|c|c|c|}
\hline 80937.40 & 3.591332 & $27.94+81$ \\
\hline 51072 & 5.473379 & 18.27024 \\
\hline 111725.3 & $5.6196+6$ & 17.79471 \\
\hline 16900.87 & 5.763270 & 17.35125 \\
\hline 86127,3 & 6.839853 & 15.06057 \\
\hline 84774.81 & 7.421858 & .13 .47371 \\
\hline 29620.75 & 8.319527 & 12.01291 \\
\hline 22758.96 & 9.198330 & $10.871+7$ \\
\hline 45936.7 & 9.003391 & 11.10692 \\
\hline 6393.37 & 9.658533 & 10.35349 \\
\hline 21525.42 & $11.2234 d$ & 8. 205219 \\
\hline 63360.78 & 10.37918 & 9.534553 \\
\hline 23922.79 & $12.243 \equiv 2$ & 8.157570 \\
\hline 33324.18 & 12.09372 & Q.293742 \\
\hline 6640.325 & 10.33887 & 9.272222 \\
\hline 33579.63 & 12.11346 & Q. $25 \leq 275$ \\
\hline 19473.72 & 12.25750 & B. 153285 \\
\hline 13122.69 & 10.8208: & $2.2+1448$ \\
\hline 34312.15 & $12.0075 \Xi$ & 6.322023 \\
\hline 9130.212 & 12.98597 & 7.700513 \\
\hline 2244.06 & 12.17591 & 8. 212932 \\
\hline 10016.49 & $13.797 \Xi 3$ & 7.247642 \\
\hline 13394.64 & 12.97049 & 7.709311 \\
\hline 13106.1 & $14.721+5$ & 8. 700553 \\
\hline $14954.9 \$$ & 13.46910 & 7. $+243 \equiv 9$ \\
\hline 64754.97 & 14.35595 & 6. $\$ 55750$ \\
\hline 18150.3 & 16.42432 & 6.092531 \\
\hline 16553.37 & 16.92998 & $5,90 \in \leq \Omega 1$ \\
\hline 16995.82 & 18.17292 & 5.502592 \\
\hline 17339.81 & 16,69475 & 5.999906 \\
\hline 4551.792 & $19.515+6$ & 5.400991 \\
\hline 10918.15 & 18.82039 & 5.313394 \\
\hline 13084.47 & 18.37282 & $5.4+2315$ \\
\hline 2413.278 & $22.08 \equiv 6=$ & 4.527013 \\
\hline 13894.58 & 21.19250 & 4.712525 \\
\hline 2634.65 & $23.38 E 61$ & 4.275550 \\
\hline 12228.93 & 21.76566 & $1.52+391$ \\
\hline 22415.77 & 22.72592 & 4. 400251 \\
\hline 9806.643 & 25.56935 & 3.995525 \\
\hline 15312.02 & 27.10102 & 3. $3 a \leq 997$ \\
\hline 4604.04 & 31.75219 & $3.1+2388$ \\
\hline 9830.016 & 25.25020 & 3.902404 \\
\hline 4708.368 & 31.19810 & 3.205322 \\
\hline 4583.629 & 27.79135 & 3.598241 \\
\hline 2777,292 & 25.53250 & 3.751872 \\
\hline 19151.49 & 32.85752 & 3.043437 \\
\hline 9250.10 & 48.73555 & 2.251995 \\
\hline 0 & ERA & 0 \\
\hline
\end{tabular}


TABLE C.5 CBA 35\% GS5

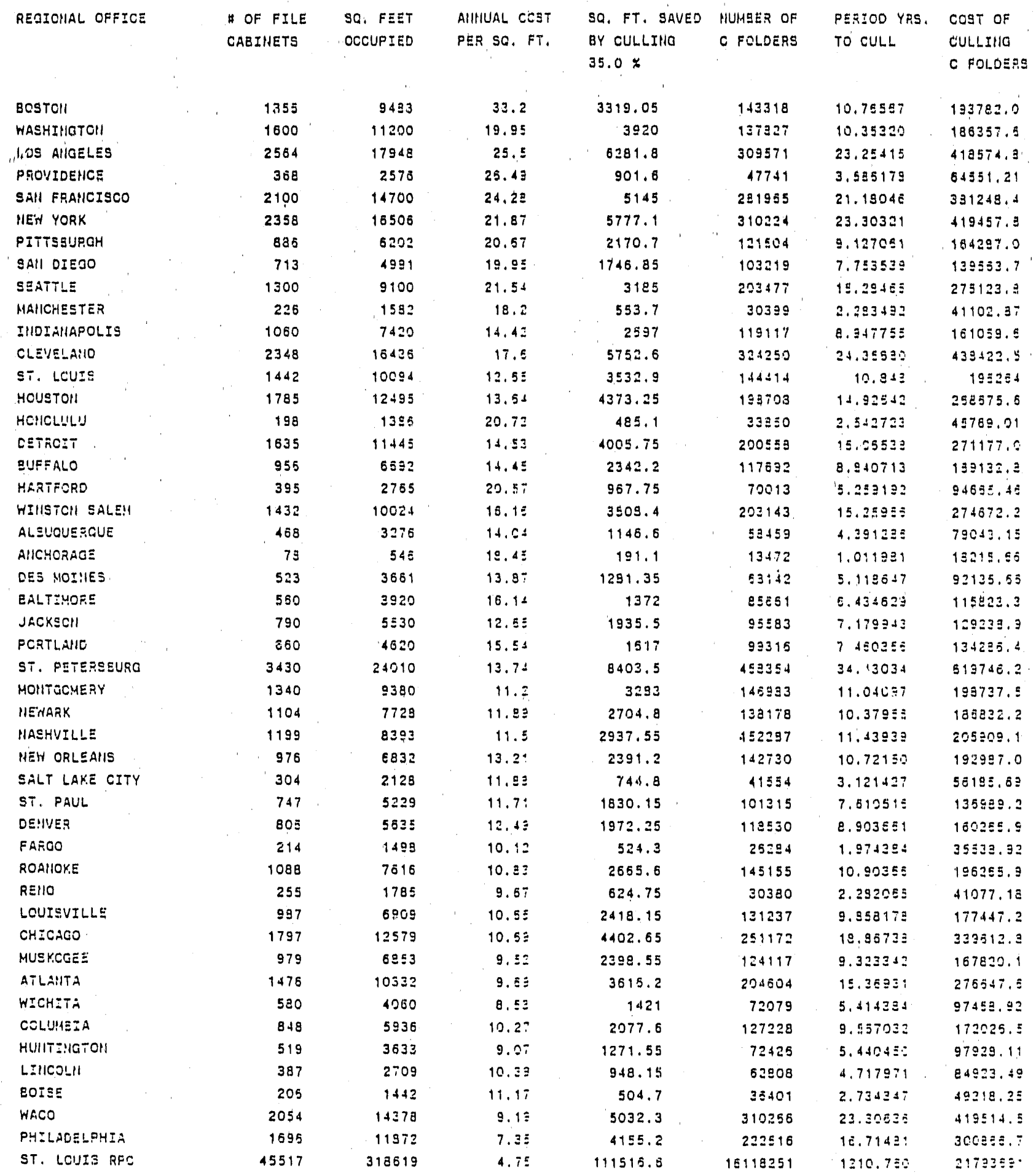


TABLE C.5 (Cont'd)

REOICIAL OFFICE

ECSTCH

WASHIIIOTOH

LOS AIIOELES

PROVIOEIICE

SAII FRAIICISCO,

NEEY YORK

PITTE ZURAH

SAII DIEDO

SEAT:LEE

MAICHESTER

IIIOI AllAPOLIS

CLESIELAIIO

ST. LCLITS

HCUETOH

Heileidul]

DETFETT

BUFF ALJ

HAPT:PRD

HIIIETOH SALEM

ALIUYUEROUE

AIICHCP.AGE

DES MCIHES

BALTEYIORE

JACKSOH

POPTLAMID

ST. PETERSBURQ

MCHTOCMERY

MENARK

HASHUILLE

MEIY COLLEAHE

SALT LAKE CITY

ST. EAUL

OE!IYE?

F גRבO

ROAIICKE

RE!IC

LOU:EVILLE

CHICAOD

MUSKESEE

ATLAITT A

HICHITA

COL_:?AE:A

HUHTEHOTOH

LIIICSLI!

cors:

WACE

PHILAOELPHIA

ST. LOUIS RFC
AIIIUAL BEHEFIT PAYEACK

OF CULLIMIO

ARP

\begin{tabular}{|c|c|c|}
\hline 110192.4 & 1.752578 & 58.88411 \\
\hline 78204 & 2.382558 & 41.93447 \\
\hline 160185.9 & 2.013056 & 39.20935 \\
\hline 23893.38 & 2.702788 & 36.99912 \\
\hline 124920.6 & 3,051928 & 32.76819 \\
\hline 128345.1 & 3.319935 & 30,12106 \\
\hline 44868.35 & 3.881534 & 27,31095 \\
\hline 34349.85 & 4.004737 & $24.970+2$ \\
\hline 68504,9 & 4.040255 & 24.93600 \\
\hline 10077.34 & 4.079742 & 24.51738 \\
\hline 37448.74 & 4.300821 & 23.25147 \\
\hline 101245.7 & 4.330290 & $23.0 \leq 319$ \\
\hline 44691.18 & 4.339193 & 22.83757 \\
\hline 59651.13 & 4.504115 & 22.20191 \\
\hline 10051.27 & $4,5 \leq 3 \leq 54$ & 21.26086 \\
\hline 58203.54 & $4.6 \equiv \geqslant 114$ & 21,16330 \\
\hline 33844.79 & $1.7018+1$ & $21.25 \pm 25$ \\
\hline 192006.51 & $4.755+77$ & 21.02338 \\
\hline $5 E 595.74$ & 4.244570 & 20.54123 \\
\hline 16092.25 & 4.910042 & 20.36542 \\
\hline 3525.735 & $5.1 \leq \pm 353$ & 19.35584 \\
\hline 17772,32 & $5.12 \leq 221$ & 19.23930 \\
\hline 22144,08 & $5.2304+1$ & 19.11894 \\
\hline 24484.07 & 5.272491 & 18.94430 \\
\hline 25129.19 & 5.344056 & 18.71237 \\
\hline 115484.0 & 5.357437 & 18.53090 \\
\hline 35789.6 & $5.40 .45+2$ & 18.50158 \\
\hline 32160.07 & $5.8094+6$ & 17.21334 \\
\hline 33791.82 & 6.095255 & 16.40517 \\
\hline $315 \mathrm{a7.75}$ & 6.109582 & 16.36780 \\
\hline $80+8.224$ & 6.349939 & $15.7+018$ \\
\hline 21431.05 & 6.392092 & 15.64432 \\
\hline $24633 .+0$ & 5.305040 & 15.371033 \\
\hline 5305,916 & $\varepsilon, \varepsilon \equiv 79 \Xi 1$ & 14.92907 \\
\hline 28858.44 & $6.73 \pm 530$ & 14.70894 \\
\hline 6041.332 & 6.799259 & 14.70727 \\
\hline 25753.29 & 6.890271 & 14.51321 \\
\hline 47064,32 & $7.2: 3928$ & 13.25222 \\
\hline 22934.19 & 7.349510 & 13.50534 \\
\hline 35040.97 & $7.3 \leqq 4975$ & 12.53929 \\
\hline 12121.13 & 8.040416 & 12.43715 \\
\hline 21336.95 & 8.052370 & 12.40329 \\
\hline 11532.95 & 8.491152 & 11.77596 \\
\hline 9851.279 & e.52055 & 11.80018 \\
\hline 5637.495 & 8.730512 & 11.45409 \\
\hline 46246.83 & 9.071206 & 11.02329 \\
\hline 30.540 .72 & 9.851329 & 10.15091 \\
\hline 329704.0 & 41.14314 & $2.4 \pm 053$ \\
\hline
\end{tabular}

AIIIUAL BEIEETT PAYEACK AFR :

CULLIHI TO RPE CULLIIIOWRPE CULLIIIO-PPC

\begin{tabular}{|c|c|}
\hline 2.052189 & $49.728+3$ \\
\hline 3.127845 & 31.97203 \\
\hline 3.211228 & 31.14074 \\
\hline 3.293297 & 30.38470 \\
\hline $3.79+202$ & 25.35600 \\
\hline 4.241052 & 23.57898 \\
\hline 4.754015 & 21.03484 \\
\hline 5,250217 & 12.02503 \\
\hline $5.14479 \vdots$ & 19.43712 \\
\hline 5,519140 & 19.11959 \\
\hline 6.413399 & 15.52239 \\
\hline 5.930967 & $13.8508 \leq$ \\
\hline 6.996224 & 14.29342 \\
\hline 8.910703 & 14.47030 \\
\hline 5.907930 & $12.925+0$ \\
\hline 6.921977 & 14.44673 \\
\hline 7.004289 & 14.27592 \\
\hline 6.183322 & 16.17253 \\
\hline 6.951514 & $1+.57+64$ \\
\hline 7.42 .0553 & $13 .+750^{\circ}$ \\
\hline $0.95788 \mathrm{a}$ & 14.37253 \\
\hline 7.884335 & 12.62337 \\
\hline 7.411705 & 13,49217 \\
\hline 8.452298 & 11.23114 \\
\hline 7.595630 & $12.9225 \%$ \\
\hline 8.203402 & 12.19006 \\
\hline 9.395220 & $10.5 \pm d 9 z$ \\
\hline 9.074274 & $10.336 \varepsilon=$ \\
\hline $10,384 \vdots 2$ & $9.5297+2$ \\
\hline 9,539957 & 10.48223 \\
\hline 10.53025 & 9.451559 \\
\hline 10.75451 & $9.292+22$ \\
\hline 10.49375 & 9.524928 \\
\hline 12.62264 & 7.922272 \\
\hline 12.11008 & 8.257595 \\
\hline 13.36377 & 7.482913 \\
\hline 12.43752 & 8.040195 \\
\hline 12.99624 & 7.700456 \\
\hline $14.858=0$ & 6. $8174 E 5$ \\
\hline 15.48629 & 6.457321 \\
\hline 18.14411 & $5.511+29$ \\
\hline 15.00011 & 0.606515 \\
\hline 17.92748 & 5.609314 \\
\hline 15.98077 & 6.290921 \\
\hline 15.19000 & 6.523277 \\
\hline 18.77576 & 5.325015 \\
\hline 27.94895 & 3.530793 \\
\hline
\end{tabular}


TABLE C.6 CBA 35\% GS9

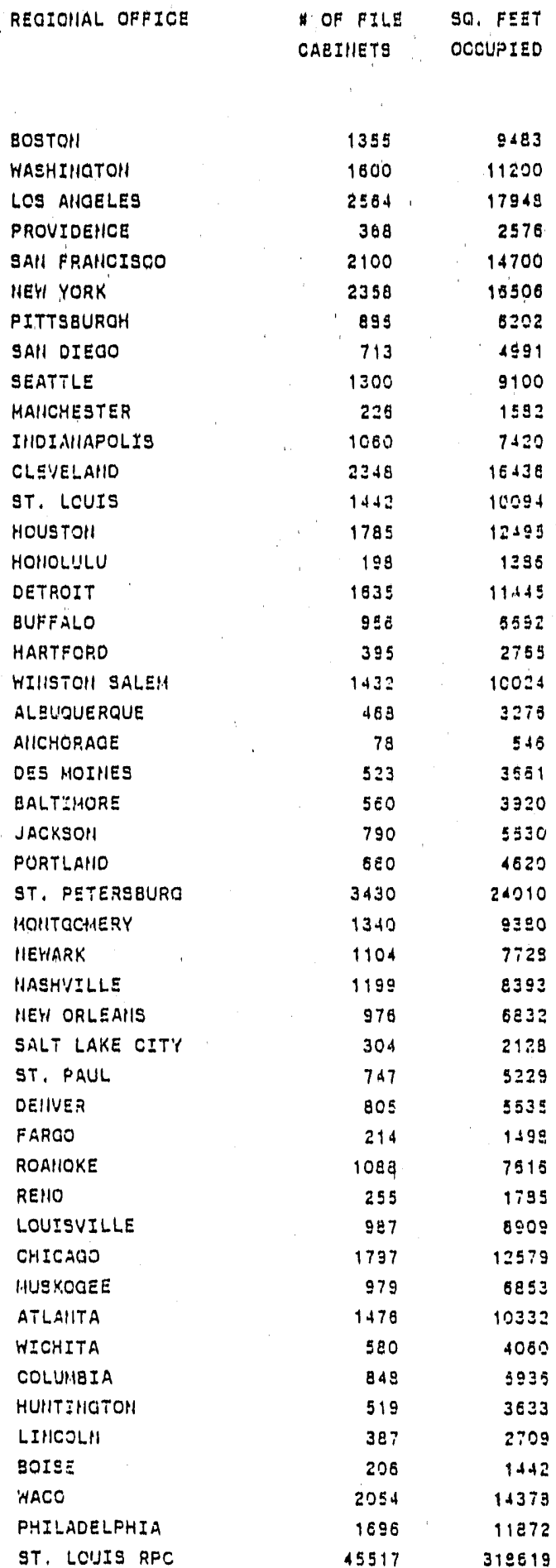

AIIIIUAL COST

PER SO, PT.
SQ, FT, SAVED HUHEEA OF BY CULLIHA O FOLDERS $35.0 \times$

\begin{tabular}{|c|c|c|}
\hline 33.2 & 3319,05 & 143318 \\
\hline 19.95 & 3920 & 137827 \\
\hline 25.5 & 6291.8 & 309371 \\
\hline 29.49 & 901.6 & 47741 \\
\hline 24.29 & 5145 & 281965 \\
\hline 21.87 & 5777.1 & 310224 \\
\hline $20.0 i$ & 2170.7 & 121304 \\
\hline 19.95 & 1748,85 & 103219 \\
\hline 21.54 & 3195 & 203477 \\
\hline 19.2 & 553.7 & 30399 \\
\hline 14,12 & 2597 & 119117 \\
\hline 17.5 & 5732.8 & $32+250$ \\
\hline 12.85 & 3332.9 & $1+t+1 d$ \\
\hline 13.84 & 4373.25 & 193708 \\
\hline 20.72 & 485.1 & 33350 \\
\hline 14.53 & 4005.75 & 200553 \\
\hline 14.45 & 2342.2 & 117892 \\
\hline 20.57 & 967,75 & 730013 \\
\hline 15.18 & 3508,4 & $2031+3$ \\
\hline 14.04 & 1140.8 & 53459 \\
\hline 18.45 & 191.1 & 134.72 \\
\hline 12.27 & 1291.35 & $031+2$ \\
\hline $15.1 \mathrm{~s}$ & 1372 & 85661 \\
\hline 12.85 & 1935.5 & 95583 \\
\hline 15.54 & 1817 & 99316 \\
\hline 13.74 & 8403.5 & 458394 \\
\hline 11.2 & 3283 & 148983 \\
\hline 11.89 & 2704.8 & 133178 \\
\hline 11.5 & 2937.55 & 152287 \\
\hline 13.21 & 2391.2 & 142730 \\
\hline 11.92 & 744.8 & 41554 \\
\hline 11.71 & $1830.1 \mathrm{E}$ & 101315 \\
\hline 12.49 & 1972.25 & 119530 \\
\hline 10.12 & 524.3 & 25294 \\
\hline 10.83 & 2665.8 & 145155 \\
\hline 9.57 & 624.75 & 30380 \\
\hline $10.8 \mathrm{E}$ & 2419.15 & 131237 \\
\hline 10.59 & 4402.65 & 251172 \\
\hline 9.52 & $23 \approx 8.55$ & $12+117$ \\
\hline 9.59 & 3616.2 & 204604 \\
\hline 8. 43 & 1421 & 72079 \\
\hline 10.27 & 2077.5 & 127228 \\
\hline 9.07 & 1271.55 & 72425 \\
\hline 10.39 & 948.15 & 62808 \\
\hline 11.17 & 504.7 & 36401 \\
\hline 9.19 & 5032.3 & 310265 \\
\hline 7.35 & 4155.2 & 292518 \\
\hline
\end{tabular}

PERIOD YRS, COEI OF TO OULL

Cullisilla

C POLOESB

$23.25415 \quad 627952.3$

$3.505179 \quad 96825.81$

$21.18046 \quad 971272.6$

$23.30321 \quad 629130.7$

$9.127001 \quad 248430.8$

$7.753539 \quad 209315.5$

15. $294 E 5 \quad 412595.7$

$2.293492 \quad 61554.30$

6.947753 241899.4

$24,35530 \quad 657033.8$

$10.943 \quad 292993$

$14.92842 \quad 403.213 .4$

$2.542723 \quad 68553.52$

$15.06339 \quad 405783$.

$8.940713 \quad 238699.2$

5.259192 141798.4

$15.25953 \quad 412009.3$

$4.391285 \quad 119504.7$

$1.011281 \quad 27323.49$

$5.119847 \quad 139203.4$

$6.43+629 \quad 173734.9$

$7.179943 \quad 193853.4$

$7.460358 \quad 201429.6$

$34.43034 \quad 929619.3$

$11.04097 \quad 298108.3$

$10.37955 \quad 290248.3$

$11.43939 \quad 308983.7$

$10.72150 \quad 289480.9$

$3.121+27 \quad 8+278.52$

$7.810516 \quad 205223.9$

8.203581 240329.3

1.974324 53309.39

$10.90355 \quad 294398.3$

$2.292035 \quad 61615.77$

$9.853179 \quad, 56170.9$

$18.86739 \quad 509419.2$

$9.323342 \quad 251730.2$

$19.35931 \quad 414971.4$

5.114334 14618a.3

$9.557032 \quad 258039.8$

$5.460+50 \quad 146892.1$

$4.717971 \quad 127385.2$

$2.734347 \quad 73827.39$

23.30535 629271.9

$15.71481 \quad 451300.0$

$1210.760 \quad 32409537$ 
TABLE C.6 (Cont'd)

REOIOIAL OFFICE

\begin{tabular}{|c|c|}
\hline HOSTOH & 110192,4 \\
\hline WASHIHOTOH & 79204 \\
\hline LOS AllaELES & 100105.9 \\
\hline PFOVIDEIICE & 23883,38 \\
\hline SAll FRAILISCO & 124920.6 \\
\hline HEIY YORK & 128349.1 \\
\hline PITTSBUKOH & $1,1800.38$ \\
\hline SAII DI $\approx 20$ & 343.19 .83 \\
\hline 9EATTLE & 68004.9 \\
\hline HAILCHESTER & 10077.34 \\
\hline IIIOIAIIAPOLIS & 37448.74 \\
\hline CLEVELAIIO & 101249.7 \\
\hline ST. LOUIS & 44891.18 \\
\hline HOUSTOII & 59851.13 \\
\hline HOHOLIJLIJ & 10051.27 \\
\hline DETFOIT & 58203,54 \\
\hline BUF F ALO & $339+4.79$ \\
\hline HARTFORD & 19905.31 \\
\hline WIHSTOH SALEH & 98099.74 \\
\hline ALQUQUEAQUE & 18098,26 \\
\hline AIICHORAOE & 3525.799 \\
\hline DES MOINES & 17772.32 \\
\hline BALTIMORE & $221+4.08$ \\
\hline$J A C K S O H$ & 24484.07 \\
\hline PORTLAHIO & 25129,18 \\
\hline BT, PETEASBURG & $11 E+64.0$ \\
\hline MOHTOONERY & 36769.6 \\
\hline MEIYARK & 32160.07 \\
\hline HASHVILLLE & 33781.82 \\
\hline HEIY ORLEAHS & 31597.75 \\
\hline SALT LAXE CITY & 8848.224 \\
\hline ST, PAUL & 21431.05 \\
\hline DEHVER & 24633.40 \\
\hline FARQO & 5305.916 \\
\hline ROAYIOKE & $29868.4 d$ \\
\hline REHO & 6041.332 \\
\hline LOUISVILLE & $257 \pm 3.29$ \\
\hline CHICACO & 17084.32 \\
\hline MUS KCOEE & $2293+, 19$ \\
\hline ATLAIITA & 35040.97 \\
\hline WICHITA & 12121.13 \\
\hline COI.U1.15IA & 21336.95 \\
\hline HUITIIIOTOH & 11532.95 \\
\hline LIIICOLII & 9851,278 \\
\hline Borss & 5037.499 \\
\hline WACO & 16240.23 \\
\hline PHILLADE!PHIA & $30 \leq 40.73$ \\
\hline ST. LOUIS RPC & 529704.0 \\
\hline
\end{tabular}

AIIIUAL BEIIEPIT PAYHACK ARR OF OULLINO

\begin{tabular}{|c|c|c|}
\hline 2.637807 & 37.90940 & $94+26.97$ \\
\hline 3.374 .192 & 27.97031 & 39984 \\
\hline 3.919985 & 25.31290 & 130347 \\
\hline 4.054149 & 24.08008 & 19600,7 \\
\hline 4.577899 & 21.84412 & 100481. \\
\hline 4,979902 & 20.08071 & 98903,95 \\
\hline 3.492302 & 18.20730 & 34557.84 \\
\hline 8.007105 & $16.0469 \mathrm{~g}$ & 28552.1 \\
\hline 6.015397 & 18.62400 & $\$ 3476.15$ \\
\hline 0.118113 & 16.34490 & 74.17 .255 \\
\hline 0.451292 & 15,90098 & 25112,99 \\
\hline 0.495420 & 15.39548 & 73920.9 \\
\hline 6.553775 & $15,2 \$ 938$ & 27909.91 \\
\hline 6.756173 & 14.90127 & 39878.19 \\
\hline 0.830331 & 14.81057 & 7747.047 \\
\hline 6.988672 & $14.3 \cos \theta$ & 39175,2 \\
\hline 7.092762 & 14.17894 & 22719.3 \\
\hline 7.133215 & 14.01892 & 15309.80 \\
\hline 7.257003 & 13.76092 & 40030.84 \\
\hline 7.385003 & 13.37761 & 10651.9 \\
\hline 7.749597 & 12.90339 & 2818.07 \\
\hline 7.775331 & 12.85953 & 11085.91 \\
\hline 7.84 .5662 & 12.74599 & 15627.08 \\
\hline 7.917737 & 12.02997 & 15290.45 \\
\hline 8.015085 & 12.17491 & 17447.43 \\
\hline 8.051155 & 12.42057 & 75547.46 \\
\hline 8.107413 & 12.33438 & 21175.35 \\
\hline 8.714170 & 11.47 .556 & 19312.2 \\
\hline 9.142997 & 10.93745 & 19828.46 \\
\hline 9.164329 & 10.91187 & 20229.55 \\
\hline 9.524909 & 10.49878 & 5310.124 \\
\hline 9.588138 & 10.42955 & 12737.84 \\
\hline 9.759000 & $10.2 \$ 689$ & 15269.2 \\
\hline 10.04897 & $9.9532+6$ & 2815,49 \\
\hline 10.19794 & $9.805 a 90$ & 16208.8 \\
\hline 10.19903 & 9.804247 & 3073.77 \\
\hline 10,33540 & $9.575+77$ & 14257.08 \\
\hline 10.82399 & 9.238919 & 26151.7 \\
\hline 11.02426 & 9.070892 & $114,11.09$ \\
\hline 11.84246 & 8.444189 & 17884.02 \\
\hline 12.05082 & $8.29144 d$ & 5371. \\
\hline 12.09356 & 8.269238 & 11468.3 \\
\hline 12.73672 & 7.851309 & 5493.095 \\
\hline 12.93083 & 7.733453 & 5347,566 \\
\hline 13.09576 & 7.836054 & 3240.1 \\
\hline $13,806 \Omega 0$ & $7.3+9 \geq 51$ & 22343. \\
\hline 14.77699 & 6.737275 & 10803. \\
\hline 81.71471 & 620359 & \\
\hline
\end{tabular}

AHIUAL BEHEFIT

CULLIIIO TO APC

PAYBACK

AAR \pm

CULLINOARPC CULLINOARC

\begin{tabular}{|c|c|}
\hline 3,078234 & 32.48562 \\
\hline 1.891468 & 21,31928 \\
\hline 4.810039 & 20.76049 \\
\hline 4.939946 & 20.24313 \\
\hline 3.691303 & 17.37086 \\
\hline 0.381593 & 15,71933 \\
\hline 7.131023 & 14,02323 \\
\hline 7.884326 & 12.68339 \\
\hline 7.717192 & 12.95308 \\
\hline 8,278785 & 12.07906 \\
\hline 9.620097 & 10.39490 \\
\hline $8.898+51$ & 11.24043 \\
\hline 10.49433 & 9.529949 \\
\hline 10,36609 & 9.949073 \\
\hline 0.861895 & $11.28+29$ \\
\hline 10.38298 & 9.831159 \\
\hline 10.50043 & 9.517975 \\
\hline 9.274984 & 10.78168 \\
\hline 10.29227 & 9.718027 \\
\hline 11.13083 & 8.984049 \\
\hline 10,43650 & 9.581754 \\
\hline 11.82950 & $8.45552,3$ \\
\hline 11.11759 & 8.994780 \\
\hline 12.67840 & $7.887+29$ \\
\hline 11.5449 .4 & 8.861799 \\
\hline 12.30510 & 8.128709 \\
\hline 14.07794 & 7.103288 \\
\hline 14.51141 & 8.391128 \\
\hline 15.57678 & 0.119808 \\
\hline 14.30978 & 0.988224 \\
\hline 15.87039 & 6.301039 \\
\hline 18.13170 & B. 198948 \\
\hline 15.74814 & 6.349952 \\
\hline 18.93395 & 5.291515 \\
\hline 18.16509 & 5.505084 \\
\hline 20.04565 & 4.988608 \\
\hline 18,85629 & 5,380123 \\
\hline 19.47936 & 5.133837 \\
\hline 22.00230 & 4.344977 \\
\hline 23.22944 & 4.304880 \\
\hline 27.21617 & 3.671296 \\
\hline 22.50017 & 4.444410 \\
\hline 25.74123 & 3.739543 \\
\hline 23.92918 & 4.197947 \\
\hline 22.78500 & 4.388851 \\
\hline 28.16364 & 3.550876 \\
\hline 41.77342 & 2,393860 \\
\hline
\end{tabular}


TABLE C.7 CBA 40\% GS5

\begin{tabular}{|c|c|c|c|c|c|c|c|}
\hline REQTOHAL OFFICE & $\begin{array}{l}\text { OP FILA } \\
\text { OABIHETS }\end{array}$ & $\begin{array}{l}\text { SQ, PEET } \\
\text { OCCUPIED }\end{array}$ & $\begin{array}{l}\text { AIIIUAL GOST } \\
\text { PER SQ, FT. }\end{array}$ & 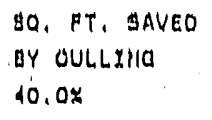 & $\begin{array}{l}\text { MUHEER OP } \\
\text { C FOLOERS }\end{array}$ & $\begin{array}{l}\text { PEAIOO YPS. } \\
\text { TO CULL }\end{array}$ & $\begin{array}{l}\text { COST CF } \\
\text { CULLIIIO } \\
\text { C FOLCERS }\end{array}$ \\
\hline BOSTOII & $135 \pi$ & 9483 & 33.2 & 3793,2 & 143310 & 10.76567 & 193792.0 \\
\hline WASHIHOTOH & 1600 & 11200 & 19,91 & 1480 & 137927 & 10.39320 & $10835 \% .0$ \\
\hline LOS AllOELES & 2984 & 17848 & 25.5 & 7179.2 & 309871 & $23,25+18$ & 018574.9 \\
\hline PROVIOEIICE & 388 & 2578 & 26.49 & 1030.4 & 47741 & 3,580173 & 04531.21 \\
\hline SAII FRAIICISCO & 2100 & 14700 & 24,39 & 8080 & 281905 & 21.10043 & $3812+8,4$ \\
\hline HEW YORK & 2398 & 16500 & 21,87 & 0802,1 & 310224 & 23,30331 & $419 d 57,9$ \\
\hline PITTSQUPAH & 880 & 0202 & 20.57 & $2480, B$ & 121904 & 9.127081 & $10+287.0$ \\
\hline SAII OIECO & 713 & 4921 & 19,28 & 1996.4 & 103219 & 7.753939 & 139383.7 \\
\hline GEATTLE & 1300 & 9100 & 21.54 & 3840 & 203177 & $15.29+83$ & 273123,9 \\
\hline "AICHESTER & 228 & 1582 & 19.2 & 832.8 & 30399 & 2.293492 & 41102.87 \\
\hline IHOIANIAPOLIS & 1090 & $7 \$ 20$ & 14,42 & 2988 & 119117 & $8.2+7735$ & 101059.9 \\
\hline CLEYELLAIIO & 2348 & $15+39$ & 17,3 & 8974.4 & 324250 & 24,35330 & $138+22.3$ \\
\hline ST. LOU:3 & 1442 & 10094 & 12.82 & 4037.8 & $1+141+$ & 10.943 & 192294 \\
\hline HOUSTOH & 1785 & $12499^{\prime}$ & 13,44 & $\$ 990$ & 198700 & 14.92012 & 280473.5 \\
\hline HOIOLULU & 199 & 1389 & 20.72 & 554.4 & 33850 & 2.542723 & 45759.21 \\
\hline DETROIT & 1035 & $11+49$ & 14.33 & 9578 & 200998 & 15.05539 & 271177.0 \\
\hline BUFFALO & 998 & 8532 & $14,4:$ & 2070.0 & 117592 & $8.840 \div 13$ & 159132.3 \\
\hline HARTFORO & 393 & 2753 & 20,37 & 1108 & 7001.3 & 5,259122 & 94085,43 \\
\hline HIIISTOH SALEH & 1432 & 10024 & 18.19 & 4009.8 & 203143 & 15.25953 & $274 d 72.2$ \\
\hline AL gUGUESOUE & 169 & 3275 & 14.04 & 1310.4 & $50+59$ & 4,391235 & 79043,13 \\
\hline AllCYORADE & 78 & 543 & 18.43 & 210.4 & $13 \$ 72$ & 1.011291 & 13215.98 \\
\hline OES MOIHES & 323 & 3681 & 13.94 & 1464,4 & .68142 & $5.1135+7$ & 92135.89 \\
\hline BALTIMORE & 500 & 3920 & 10.11 & 1960 & 85561 & $0.13+223$ & $11 \leq 823.3$ \\
\hline JACXSON & 790 & 5930 & 12,95 & 2212 & 23503 & 7.179943 & 129232.9 \\
\hline PORTLAMO & 880 & 4622 & 15.91 & 1848 & 99316 & 7.460393 & 1342964 \\
\hline ST, PETERSBURO & 3430 & 24010 & 13.74 & 9604 & 199354 & 3.4 .43034 & $6197+4.2$ \\
\hline MOIITOCHERY & 1340 & 9380 & 14.2 & 3752 & 148983 & 11.04097 & 198737.5 \\
\hline HE'IARK & 1104 & 7729 & 11.28 & 3091.2 & .138179 & 10.37953 & 185332.2 \\
\hline NAEHVILLE & 1199 & 8393 & 11.5 & 3397.2 & 132287 & 11.43939 & 205900.1 \\
\hline MEH ORLEAIIS & 978 & 6832 & 13.21 & 2732.8 & 142730 & $10,721 \leq 0$ & 192987.0 \\
\hline SALT LAKE CITY & 304 & $212 \theta$ & 11.83 & 851.2 & 41554 & $3.121+27$ & 50185.59 \\
\hline ST. PAUL & 747 & 5229 & 11.71 & 2091.6 & 101315 & 7.010915 & 135929,2 \\
\hline DEIIVEa & 805 & 5935 & 12.17 & $2.2 \div 4$ & 118530 & 0,903881 & 160255.9 \\
\hline PARQO & 214 & 1499 & 10.12 & 599.2 & 29284 & $1.97+334$ & 35538.92 \\
\hline ROAPIOKE & 1080 & 7816 & 10.83 & 30.40 .4 & 145155 & 10.80350 & 198235.9 \\
\hline REHO & 295 & 1785 & $9.5:$ & 714 & 30380 & 2.2922065 & 41077.18 \\
\hline LOUISVI!LLE & 987 & 6969 & 10.9. & 2763.8 & 131237 & 9.850178 & 177447.2 \\
\hline CHICACO & 1797 & 12579 & 10.99 & 5031.8 & 291172 & 18.96738 & $339: 12,8$ \\
\hline MUSKCOEZ & 979 & $68: 3$ & 9,52 & 2741.2 & 124117 & 9,323342 & 167820.1 \\
\hline ATLANITA & 1476 & 10332 & 9.53 & 4132,8 & 204804 & 15,36931 & $2785 \times 7.5$ \\
\hline WICHITA & 580 & 4050 & e. 53 & 1624 & 72079 & 5.414394 & 97492,32 \\
\hline COLUNEIA & $8+8$ & 5936 & 10.27 & 2374.4 & 127228 & 9.557032 & 172025.5 \\
\hline HUIITIMATCH & 513 & 3833 & 9.67 & 1453.2 & 72426 & $5.440+50$ & 97929.11 \\
\hline LINCOLHI & 387 & 2709 & 10.37 & 1083.6 & 62908 & 4.717971 & 84923.49 \\
\hline BOISE & 208 & $14+2$ & 11.17 & 578.8 & 36401 & 2.734347 & 49218,25 \\
\hline WACO & 2054 & 14378 & 9.19 & 5751.2 & 310280 & 23.30833 & 419514,5 \\
\hline PHILA,DELPHIA & 1096 & 11972 & 7.35 & 4748.9 & 222510 & $19,71+81$ & 300958.7 \\
\hline ST, LOUIS RPC & 45517 & 318019 & 4,75 & 127447.0 & 16119251 & 1210.75 & 21793821 \\
\hline
\end{tabular}


TABLE C.7 (Cont'd)

REQLOIIAL OPFICE

BOSTON

WASHIIITTON

LOS AllOELAE

PHOVTDEHCE

SAII FRAIICISCO

IIEIY YORK

PIYTSEUPOOH

SAII OIEMO

SEATTLE

MAIICHESTER

IIIOTAIIAPOLIS

CLUUELAIIO

ST. LQUT3

Houstoll

HOHOLULU

OETROIT

BUPFALO

HARTEERO

WHISTCII SAL EI

ALgUGUgRQUE

AIICYORAOE

OES MCIILE

AALTEIORE

JACKSGH

PORTLAIIO

ST. PETERSBUPO

MOHTOCHERY

MEHARK

HASHUILLE

NEIY OPLEAHY

SALT LAKE CITY

ST. PAUL

OEIIVEA

\section{FARCO}

ROAIIOKE

REIIO

LOUISVILLI

CHICAOO

MUSKCOE:

ATLAHTA

HICHITA

COLUMEIA

HUIIT IHQTOII

LIIICCLII

HOISE

WACO

PHILADELPHIA

ST. LOUTS RPC
AHIIUAL GEHIEFIT PAYBACY ARR OF CULLIIHO

\begin{tabular}{|c|c|}
\hline 1.530750 & 64.98795 \\
\hline 2.088097 & 17,95039 \\
\hline $2,298+24$ & 13.73610 \\
\hline 2.384930 & 13.28471 \\
\hline 2.070433 & $37,4.470 \%$ \\
\hline 2,801213 & 34,42007 \\
\hline $3.2039+3$ & 31.21291 \\
\hline 3.504143 & 28.53793 \\
\hline 3,908981 & 28,49829 \\
\hline 3,968898 & $28.0198 \mathrm{~d}$ \\
\hline 3,703201 & 29.37311 \\
\hline 3.788999 & 29.39221 \\
\hline 3.323031 & 29.19722 \\
\hline 3,941101 & 25.37391 \\
\hline 3,984360 & 25.09813 \\
\hline 4.078719 & 24,92919 \\
\hline 4.114111 & 24.30090 \\
\hline 1.101042 & 24.03243 \\
\hline d.239087 & 23.93998 \\
\hline 4.290298 & 23.27591 \\
\hline 4,520929 & 22,12098 \\
\hline 4,536193 & $22.04+91$ \\
\hline 4. 379935 & $21.8 \$ 010$ \\
\hline 4.518880 & 21.53120 \\
\hline 4.8750 .49 & 21.39937 \\
\hline 4.699507 & 21.29241 \\
\hline 4.729324 & $21.14+86$ \\
\hline 5.083215 & 19.87339 \\
\hline 5. 333357 & $18,7+991$ \\
\hline 5.345359 & $12,7080 B$ \\
\hline 3.553197 & 17.99792 \\
\hline 5,593000 & 17,87223 \\
\hline 9.69579 & 17.58000 \\
\hline 5.980734 & 17.08270 \\
\hline 3.948901 & 15.81010 \\
\hline 5.949433 & 15.90830 \\
\hline 8.029987 & 16.98553 \\
\hline 6.313937 & 19.93797 \\
\hline 0.430921 & 15.93211 \\
\hline 0.928123 & 14.47575 \\
\hline 7.035391 & 14.21390 \\
\hline $7.05+59:$ & 14.17510 \\
\hline 7,129793 & 13,45938 \\
\hline 7.542999 & 13.25734 \\
\hline 7.839193 & 13.09037 \\
\hline 7.937305 & 12.59873 \\
\hline 8.619913 & 11.80104 \\
\hline 36.00025 & 2.777798 \\
\hline
\end{tabular}

AHIIUAL BUHEFTT OULLIIHO TO RPO

PAYBAOK ARR OULLIIIOARP CULLIHO-RPC

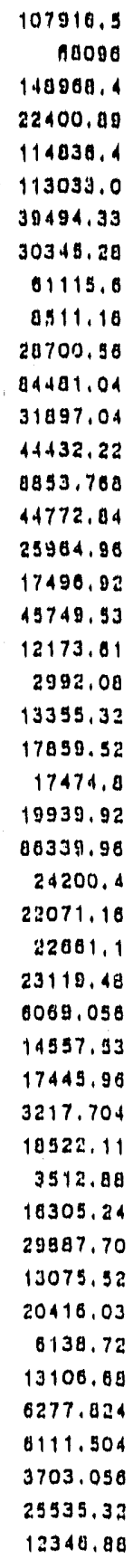

\begin{tabular}{|c|c|}
\hline 1.795800 & 39.88903 \\
\hline 2.730609 & 38,91040 \\
\hline 2,809023 & 35,90942 \\
\hline 2.801035 & 34.70251 \\
\hline 3.319940 & 30.13114 \\
\hline 3,710929 & 29.24742 \\
\hline 4,159763 & 24.03983 \\
\hline 4.599190 & 21.71295 \\
\hline 4,501095 & 22.21383 \\
\hline 1,829291 & 25,76697 \\
\hline 9.611723 & 17.81933 \\
\hline 1.189996 & 19.29931 \\
\hline 0,121090 & 19.33214 \\
\hline 0.0 .46804 & $19.937+9$ \\
\hline 3,169439 & $13 \cdot 3+4+3$ \\
\hline 0.056730 & $15.310 \mathrm{~s}$ \\
\hline 6.129793 & 19,31853 \\
\hline 9.410 .107 & $19,48 \geq 99$ \\
\hline 0.003829 & 14.95004 \\
\hline 6.492909 & 13,10122 \\
\hline $6: 097959$ & 15.42535 \\
\hline 0.898794 & $1+1+9529$ \\
\hline $0,48 \leq 242$ & 15.11962 \\
\hline $7.39 .73+$ & 13.92130 \\
\hline 0.734551 & $1+, 3+8 \div 9$ \\
\hline 7.177977 & $13.931: 0$ \\
\hline 0.212100 & 12.17709 \\
\hline 8.464990 & 11.91338 \\
\hline $9,08 B 460$ & 11.00533 \\
\hline 0.347375 & 11.97981 \\
\hline 9.257731 & 10.80179 \\
\hline 9.110197 & 10.92978 \\
\hline $9.180+19$ & 10.83533 \\
\hline 11,04481 & 9.054025 \\
\hline 10.99830 & 9.437253 \\
\hline 11.69330 & 8.951900 \\
\hline 10.88293 & 9.199793 \\
\hline 11.36298 & 8.300322 \\
\hline 12.83480 & 7.791390 \\
\hline 13.55051 & 7.379755 \\
\hline 15.07609 & $6.2937 \div 8$ \\
\hline 13.12510 & 7.618989 \\
\hline 19.90905 & $0.1106+5$ \\
\hline 13,89537 & $7.195+32$ \\
\hline 13,29125 & $7,5237+5$ \\
\hline 16.12879 & $9.08597 \mathrm{~d}$ \\
\hline 24.35783 & 4. 123770 \\
\hline ERT & 0 \\
\hline
\end{tabular}


TABLE C.8 CBA 40\% GS9

\begin{tabular}{|c|c|c|c|c|c|c|c|}
\hline AEDIOHAL OPPICE & $\begin{array}{l}\text { OF P\$LB } \\
\text { OABIIIBTS }\end{array}$ & $\begin{array}{l}\text { 9Q. PEET } \\
\text { OCCUPIEO }\end{array}$ & $\begin{array}{l}\text { AIIIUAL COST } \\
\text { PUA SO, PT. }\end{array}$ & $\begin{array}{l}\text { 9Q. PT. SAVED } \\
\text { OY CUhLIIIO } \\
\text { 10.0\% }\end{array}$ & $\begin{array}{l}\text { HUMAEA OF } \\
\text { C rOLOERS }\end{array}$ & $\begin{array}{l}\text { PERLOO YRI. } \\
\text { TO CULL }\end{array}$ & $\begin{array}{l}\text { cost of } \\
\text { CULLIIIO } \\
\text { C roLDEgS }\end{array}$ \\
\hline Boston & 1359 & 9493 & 39,2 & 3703,2 & 143910 & 10,78987 & 200873.1 \\
\hline WASHRHOTOH & 1800 & 11200 & 19.99 & 1480 & 127027 & 10,39320 & 279338.4 \\
\hline LCS AllaELES & 2504 & 17918 & 29.3 & 7179.2 & 309971 & 23,29119 & 027802,3 \\
\hline PROVIDAMICE & 308 & 2976 & 20.49 & 1030.4 & 47741 & 3,880178 & 80028.81 \\
\hline SAII PRANCISCO & 2100 & $1+700$ & 24.20 & 8000 & 291908 & 21,10018 & $0 \% 1872.8$ \\
\hline HEHI YONK & 2310 & 10900 & 21.07 & 0802,4 & 310294 & 23,30321 & 029100.7 \\
\hline PITTSGURAH & 800 & 0202 & 20.07 & 2480,8 & 121904 & 0.127001 & $240,130,3$ \\
\hline SAll OIEOO & 713 & 1991 & 19.91 & 1900.1 & 103219 & 7,793339 & $2093+9.3$ \\
\hline SEATTLE & 1300 & 9100 & 21.91 & 3640 & 203477 & 19.28485 & 413985.7 \\
\hline MAHCHESTER & 225 & 1932 & 18.2 & 032.0 & 30399 & 2,285192 & 01854,30 \\
\hline IHOIAIIAPOLIS & 1080 & 74.0 & $1+12$ & 2968 & 119117 & 0.947793 & $2+1989,+$ \\
\hline CLEVELAIIO & $23+8$ & $18+34$ & 17.6 & 8974,1 & $32+290$ & 24,39390 & 097339.9 \\
\hline 9T, LouIg & $14+2$ & 10094 & 12.03 & 1037.6 & $1+4 d 1 d$ & 10.819 & 292993 \\
\hline HCUSTOH & 1798 & $12+94$ & 13.84 & 1998 & 199708 & $14.923+2$ & 103013.1 \\
\hline HOHOLULy & 199 & 13 : & 20,72 & 594,1 & 33850 & $2.9427: 3$ & 09833,52 \\
\hline OETROLT & 1635 & $11+49$ & 14.93 & 1379 & 220958 & 15,06338 & 400763,5 \\
\hline BUPFALO & 996 & 8992 & 14.49 & 2870.8 & 1,7092 & 0.940713 & 238899,2 \\
\hline HAPTFORO & 395 & 2793 & 20.57 & 1108 & 70013 & 3.299192 & $1+1998.1$ \\
\hline WIIISTOII SALEH & $1+32$ & 10224 & 19.16 & 1009,8 & $2031+3$ & 15.25934 & 012002.3 \\
\hline AL RLVUERGUE & 189 & 3275 & 14.24 & 1310.4 & $53+9.9$ & 1,391239 & $11958 d .7$ \\
\hline AIICHORAOE & 78 & 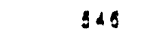 & 18.43 & 218,4 & $13+72$ & 1.011231 & $2.7323,49$ \\
\hline DES MOIHES & 523 & 3391 & $13.8:$ & 1484,4 & $881 * 2$ & 9,118947 & 139203,4 \\
\hline BALTIHORE & 980 & 3220 & 18.14 & 1988 & 83001 & 0.431529 & 173734.9 \\
\hline JACKSCHI & 790 & 5330 & 12.35 & 2212 & 99583 & 7.179943 & 193898,4 \\
\hline POPTI.AIID & 080 & $d: 20$ & 13.54 & $18 d 8$ & 99318 & 7.460393 & $201+29.8$ \\
\hline ST. PETERSEURO & 3430 & 24010 & 13.74 & 960.1 & 138334 & 34.43034 & 929319.3 \\
\hline NOIIIOOME, & 1340 & 2380 & 11,2 & 3712 & 140983 & 11.04007 & 298108,3 \\
\hline IIEHARK & 1104 & 7729 & 11.99 & 3091.2 & $\cdot 138178$ & $10,379: 9$ & 280248,3 \\
\hline MASHVILLEE & 1198 & 8393 & 11,9 & 3957.2 & 132207 & 11,43939 & 308993.7 \\
\hline MEYH ORLEAIIS & 976 & 6232 & 13.21 & 2732.8 & 142730 & 10.72150 & $299+90.5$ \\
\hline SALT LARE CITY & 304 & 2129 & 11.98 & 851.2 & 41554 & 3.121427 & $8+270.83$ \\
\hline ST, PAUL & $7 \$ 7$ & 9227 & 11.71 & 2091.8 & 101315 & 7.310516 & $20:+83.9$ \\
\hline OEIVER & 805 & 3833 & 12.49 & 2254 & 118930 & 0.903691 & 240398.9 \\
\hline FAP,QO & 214 & 1493 & 10.12 & 599.2 & 25284 & 1.974394 & 33309,39 \\
\hline AOAIIOKE & 1083 & 7518 & 10.83 & 3040.4 & 145195 & 10.90388 & 294393.9 \\
\hline REIIO & 259 & $178:$ & 9.57 & 914 & 30380 & 2.292009 & 61815.77 \\
\hline LOU1SVILLE & 987 & 6902 & 10.65 & 2783,6 & 131237 & 9.858173 & 258170.3 \\
\hline $\mathrm{CH}=\mathrm{C}=0 \mathrm{O}$ & $179 \%$ & 12579 & 10.59 & 5031.0 & 251172 & 18.88738 & $309+19.2$ \\
\hline MUS KOOEE & 979 & 6953 & 4.52 & $2 \% 41.2$ & 124117 & 9.323342 & 291730.2 \\
\hline ATLAIITA & $1+76$ & 10332 & 9.69 & 1132.8 & 224004 & 19.35931 & 114971.4 \\
\hline WICHITA & 500 & 1050 & 8.53 & 1824 & 72279 & 5.414394 & 148184.3 \\
\hline COLUMEIA & 848 & 9935 & 10.27 & 2374.1 & 127228 & 9,557032 & 252039.0 \\
\hline HUIITIIOTOH & 519 & 3533 & 9.07 & 1453,2 & 72428 & $5,440+50$ & 140892.1 \\
\hline LIHCOLH & 397 & 2709 & 10.37 & 1083.3 & B2008 & 4.717971 & 127385.2 \\
\hline BOI9E & 200 & $14+2$ & 11.17 & 575.8 & 38.101 & 2.734347 & 72827.39 \\
\hline WACO & $203 \mathrm{~A}$ & 14373 & 9.19 & 5781.2 & 310286 & 23.30639 & $8292 ? 1.8$ \\
\hline PHILAOELPHIA & 1695 & 11972 & 7,39 & 4748.8 & 222515 & 18.71481 & $4 \leq 1 \geq 00.2$ \\
\hline ST. LOUIS RFC & $\$ 5517$ & $3198 \div 9$ & 1.72 & $127 A 47,5$ & 19118251 & $1210.73 ?$ & 32590537 \\
\hline
\end{tabular}


TABLE C.8 (Cont'd)

\begin{tabular}{|c|c|c|c|}
\hline ABOTOIIAL OFPICE & 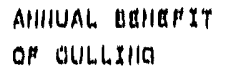 & PAYBAOK & Ann \\
\hline aostoll & 129031.2 & 2.300134 & 43,32403 \\
\hline WASHIHQTOH & 09370 & 3.127048 & 31,97293 \\
\hline LOO A1IQELES & 189008.0 & 3.102037 & 20.18780 \\
\hline PAOVIDEICE & 27290,29 & 3.8 .17301 & 28.18901 \\
\hline OAll KRALIOISOO & 1411780.4 & 4,005893 & 24,00071 \\
\hline MEIY YORK & 141994,4 & 1.317114 & $23.8+138$ \\
\hline PITTERUPGK & 11278.13 & 1,805701 & 20.80994 \\
\hline SAll OIBUOO & 39029,10 & 0.290217 & 19.02900 \\
\hline SEATTLE & 70105.0 & $0.20307: !$ & 18.99830 \\
\hline MAILCHESTER & 11910.90 & 3,933349 & 18. 07999 \\
\hline IIIOIAIIAPOLIS & 12790,28 & $1.04+002$ & 17.11541 \\
\hline CLE'JELAIIO & 119709,1 & 8.083493 & 17.98481 \\
\hline 9T, LOULS & 91075.64 & 5.734553 & 17.43014 \\
\hline HOUSTOII & 08172.79 & 0.911082 & 10.91974 \\
\hline HOHOLILU & 11487,18 & $3,9703+10$ & 10.73290 \\
\hline DETROIT & $\cos 18.31$ & 8.115089 & 16.39299 \\
\hline HUPPALO & 38073.78 & 0.171187 & 10.20430 \\
\hline HAATPQAE & 92790.43 & 0.241903 & 10.09195 \\
\hline WIISTCH SALEH & 04795.13 & 0.350530 & 13.72905 \\
\hline AL BUQUEROUR & 18380.01 & $0.44+430$ & 15.81757 \\
\hline AIICHORARE & 1049.40 & 0.780080 & $14.7+730$ \\
\hline DES HOIHES & $20311.2 \mathrm{~d}$ & 0.804390 & 14.89890 \\
\hline BALTIHORE & 25307.52 & 0.061954 & $14.5687 \mathrm{~J}$ \\
\hline JACKSOH & 27981.8 & 0.929020 & $14,+3 d 13$ \\
\hline PORTLAIID & 29717.92 & 7.014074 & 14.25704 \\
\hline ST. PETERSBURA & 131998.9 & 7.044781 & $14.1919 d$ \\
\hline HOITOCHERY & 12022,4 & 7.093997 & 14.08344 \\
\hline HEYARKK & 30754.36 & 7.021800 & 13.11192 \\
\hline MASHVILLE & $3060 \%, 8$ & 0.000035 & 12,19994 \\
\hline HEIY ORLEAHIS & 30100,29 & 0.018709 & 12.17071 \\
\hline SALT LAKE OITY & 10112.28 & $0.33+290$ & 11.99981 \\
\hline ST, PAUL. & $24+92,93$ & 8.389621 & 11.91948 \\
\hline DRIVER & 25152.40 & 0.939179 & 11.71072 \\
\hline FAROO & 0093.904 & 9. 791101 & 11.37313 \\
\hline ROAIIOKE & 32992.51 & 0.923202 & 11.20073 \\
\hline AEHO & $690 d, 38$ & 0.924157 & 11.20593 \\
\hline L.OUISVILLE & 29432.34 & 9.0 .13481 & 11.29708 \\
\hline CHICAOO & 93797.90 & 9.170926 & 10,59995 \\
\hline HUSKCOEE & 28090.22 & 9.816232 & 10.3807 .1 \\
\hline ATLAIITA & 40046.93 & 10.36215 & 9.550501 \\
\hline WICHITA & 13852.72 & 10.55304 & 9.475835 \\
\hline COLUHAEIA & 24385.08 & 19.30187 & 9.150123 \\
\hline HUHT:HDTOH & 13180.93 & 11.14483 & 0.972924 \\
\hline LIHCOLLII & 11250.00 & 11.31447 & 0.830232 \\
\hline Borag & $6+42.05 \theta$ & 11.45879 & 0.728919 \\
\hline WACO & 9.2893 .97 & 11,90999 & 8.399190 \\
\hline PHILLADELPHIA & 34903.99 & 12.92907 & $7.73+029$ \\
\hline ST, LOUIS RPC & 805370.1 & 34.00037 & 1.9391938 \\
\hline
\end{tabular}

AHIIUAL BEMGEIT PAYOACK ARR $x$ GULLIIIO TO APG OULLIHGaAPO CULLIHOARPO

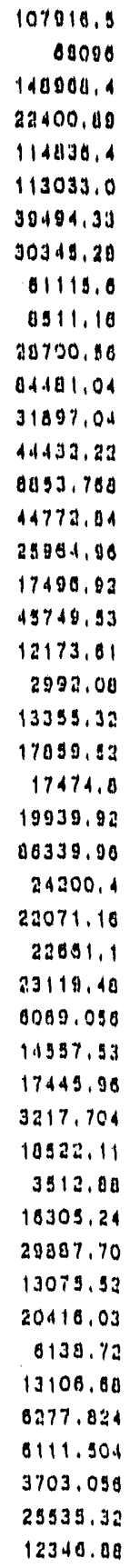

\begin{tabular}{|c|c|}
\hline $2.003+20$ & $37,129+2$ \\
\hline $1,10803.1$ & 24,38032 \\
\hline 1.214734 & 23,72243 \\
\hline 4.งล24. & 23,13901 \\
\hline 1,079000 & 20.08079 \\
\hline 9.800391 & $17.90+81$ \\
\hline 0.239049 & 10.03895 \\
\hline 0,090708 & 14,10930 \\
\hline $0,75: 9 d y$ & 14.00913 \\
\hline 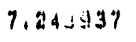 & $13.00 .10 \mathrm{~d}$ \\
\hline 0.117983 & 11.87099 \\
\hline 7,701398 & 12.94021 \\
\hline $9,1025 d 4$ & 10.02232 \\
\hline 0.070298 & $11.02+98$ \\
\hline 7.754151 & 12.89930 \\
\hline 9,009009 & 11.007 .23 \\
\hline 0.183130 & 10.91709 \\
\hline 0.118011 & 12,32183 \\
\hline 9,005739 & $11,10+03$ \\
\hline 9.739403 & $10,294+3$ \\
\hline 0.131930 & 10.91997 \\
\hline 10.34019 & 2,053024 \\
\hline 9.727004 & $10.2791 \mathrm{~d}$ \\
\hline 11.09380 & $0.01+204$ \\
\hline 10,10182 & 9.899199 \\
\hline 10.70098 & 9.297987 \\
\hline 12.31824 & $0.1190+1$ \\
\hline 12,00748 & $7.87357 \mathrm{~d}$ \\
\hline 13.02989 & 7.338023 \\
\hline 12.52100 & $7.9009+2$ \\
\hline 13.80059 & 7.201193 \\
\hline 11,11529 & $7.00+312$ \\
\hline 17.77962 & 7.297089 \\
\hline 18.56721 & 0.030017 \\
\hline 15.89445 & 0.201002 \\
\hline 17.53999 & 5.701207 \\
\hline 16.32425 & 0.129993 \\
\hline $17.041+14$ & 9.087014 \\
\hline 19.25202 & 3.194250 \\
\hline 20,32570 & 4.019333 \\
\hline 23.01414 & 4.129184 \\
\hline 19.08705 & 5.079325 \\
\hline 231.39058 & 4.273793 \\
\hline 20.84351 & $4.79798 \mathrm{~d}$ \\
\hline 19.93687 & 3.015330 \\
\hline 24.04318 & 4.057918 \\
\hline $36.5517 \mathrm{t}$ & 2.738347 \\
\hline EgR & 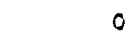 \\
\hline
\end{tabular}


$39 / 40$

APPENDIX D:

SPREADSHLET ON CLAIM FOLDER THICKNIASSES 
APPENDIX

SPREADSHEET ON CLAIM FOLDER THICKNESSES

\begin{tabular}{|c|c|c|c|c|c|}
\hline \multirow[b]{2}{*}{ File No. } & \multirow{2}{*}{$\begin{array}{c}\text { Culling } \\
\text { No. }\end{array}$} & \multicolumn{4}{|c|}{ Size (int) } \\
\hline & & Total & Right & Center & Left \\
\hline $\begin{array}{l}349-30-6229 \\
4422330 \\
\operatorname{css} 340-54-4007 \\
\text { Xss } 355-30-7900 \\
\text { XC } 26-254-801\end{array}$ & $\begin{array}{l}1 \\
2 \\
3 \\
4 \\
5\end{array}$ & $\begin{array}{l}0.375 \\
0.75 \\
0.281 \\
0.062 \\
0.843\end{array}$ & $\begin{array}{l}0.031 \\
0.031 \\
0.125 \\
0 \\
0.031\end{array}$ & $\begin{array}{l}0.312 \\
0.562 \\
0.093 \\
0.031 \\
0.656\end{array}$ & $\begin{array}{l}0.031 \\
0.156 \\
0.062 \\
0.031 \\
0.156\end{array}$ \\
\hline $\begin{array}{l}23-501-003 \\
23-487-112 \\
\text { XC } 4-116-545 \\
\text { SS } 356-48-1645 \\
29-609046\end{array}$ & $\begin{array}{r}6 \\
7 \\
8 \\
9 \\
10\end{array}$ & $\begin{array}{l}0.468 \\
0.5 \\
0.156 \\
0.625 \\
0.531\end{array}$ & $\begin{array}{l}0.125 \\
0.093 \\
0 \\
0.281 \\
0.062\end{array}$ & $\begin{array}{l}0.218 \\
0.312 \\
0.125 \\
0.156 \\
0.312\end{array}$ & $\begin{array}{l}0.125 \\
0.093 \\
0.031 \\
0.187 \\
0.156\end{array}$ \\
\hline $\begin{array}{l}153-747 \\
C 3159148 \\
\text { XC } 13-229-149 \\
\text { XC } 21878533 \\
23-491-333\end{array}$ & $\begin{array}{l}11 \\
12 \\
13 \\
14 \\
15\end{array}$ & $\begin{array}{l}1.75 \\
0.562 \\
1.5 \\
0.625 \\
0.625\end{array}$ & $\begin{array}{l}0.062 \\
0.031 \\
0.062 \\
0.062 \\
0.156\end{array}$ & $\begin{array}{l}1.375 \\
0.406 \\
1.187 \\
0.343 \\
0.281\end{array}$ & $\begin{array}{l}0.312 \\
0.125 \\
0.25 \\
0.062 \\
0.187\end{array}$ \\
\hline $\begin{array}{l}\text { XC } 1961735 \\
\text { SS } 156-44-6535 \\
\text { XC } 21600837 \\
\text { C225 } 21339 \\
\text { CSS } 356-44-2318\end{array}$ & $\begin{array}{l}16 \\
17 \\
18 \\
19 \\
20\end{array}$ & $\begin{array}{l}0.875 \\
0.406 \\
0.25 \\
1.187 \\
0.406\end{array}$ & $\begin{array}{l}0.062 \\
0.093 \\
0.031 \\
0.062 \\
0.187\end{array}$ & $\begin{array}{l}0.593 \\
0.218 \\
0.156 \\
1 \\
0.156\end{array}$ & $\begin{array}{l}0.218 \\
0.156 \\
0.062 \\
0.125 \\
0.062\end{array}$ \\
\hline $\begin{array}{l}\text { C } 29-571-122 \\
\text { C } 28-207-123 \\
\text { C } 5425626 \\
1.0-903-026 \\
14670731\end{array}$ & $\begin{array}{l}21 \\
22 \\
23 \\
24 \\
25\end{array}$ & $\begin{array}{l}1.125 \\
5 \\
0.5 \\
0.937 \\
0.343\end{array}$ & $\begin{array}{l}0.218 \\
0.218 \\
0.031 \\
0.062 \\
0.031\end{array}$ & $\begin{array}{l}0.625 \\
4.625 \\
0.312 \\
0.656 \\
0.25\end{array}$ & $\begin{array}{l}0.281 \\
0.156 \\
0.156 \\
0.218 \\
0.062\end{array}$ \\
\hline $\begin{array}{l}X C 16-206-932 \\
10001-193 \\
21265483 \\
\text { SS } 353-32-8181 \\
\text { XC } 28101-959\end{array}$ & $\begin{array}{l}26 \\
27 \\
28 \\
29 \\
30\end{array}$ & $\begin{array}{l}0.125 \\
0.781 \\
0.531 \\
0.468 \\
0.937\end{array}$ & $\begin{array}{l}0 \\
0.031 \\
0.093 \\
0.218 \\
0.093\end{array}$ & $\begin{array}{l}0.093 \\
0.312 \\
0.312 \\
0.093 \\
0.562\end{array}$ & $\begin{array}{l}0.031 \\
0.437 \\
0.125 \\
0.156 \\
0.281\end{array}$ \\
\hline $\begin{array}{l}27338 \quad 375 \\
21170068 \\
X C 6111973 \\
302-46-3666 \\
347-52-6752\end{array}$ & $\begin{array}{l}31 \\
32 \\
33 \\
34 \\
35\end{array}$ & $\begin{array}{l}0.718 \\
0.812 \\
0.968 \\
0.312 \\
0.687\end{array}$ & $\begin{array}{l}0.25 \\
0.062 \\
0.031 \\
0.125 \\
0.218\end{array}$ & $\begin{array}{l}0.312 \\
0.687 \\
0.812 \\
0.062 \\
0.406\end{array}$ & $\begin{array}{l}0.156 \\
0.062 \\
0.125 \\
0.125 \\
0.062\end{array}$ \\
\hline
\end{tabular}




\begin{tabular}{|c|c|c|c|c|c|}
\hline \multirow[b]{2}{*}{ File No. } & \multirow{2}{*}{$\begin{array}{l}\text { Culling } \\
\text { No. }\end{array}$} & \multicolumn{4}{|c|}{ Size $\left(i \pi_{0}\right)$} \\
\hline & & Total & Right & Center & Left \\
\hline $\begin{array}{l}26889788 \\
22345396 \\
\text { XC } 7-622-593 \\
\text { SS } 337-54-4463 \\
\text { XXS } 350 \quad 18 \quad 7892\end{array}$ & $\begin{array}{l}36 \\
37 \\
38 \\
39 \\
40\end{array}$ & $\begin{array}{l}0.218 \\
1.062 \\
2.031 \\
0.281 \\
0.312\end{array}$ & $\begin{array}{l}0.062 \\
0.062 \\
0.125 \\
0.125 \\
0\end{array}$ & $\begin{array}{l}0.093 \\
0.718 \\
1.562 \\
0.062 \\
0.25\end{array}$ & $\begin{array}{l}0.062 \\
0.281 \\
0.343 \\
0.093 \\
0.062\end{array}$ \\
\hline $\begin{array}{llll}X C & 19 & 866 & 384 \\
X C & 15840788 \\
344-42 & -3187 \\
27 & 074 & 037 \\
13836187\end{array}$ & $\begin{array}{l}41 \\
42 \\
43 \\
44 \\
45\end{array}$ & $\begin{array}{l}1.062 \\
0.5 \\
0.468 \\
0.781 \\
0.781\end{array}$ & $\begin{array}{l}0.125 \\
0.031 \\
0.187 \\
0.312 \\
0.031\end{array}$ & $\begin{array}{l}0.531 \\
0.312 \\
0.156 \\
0.187 \\
0.687\end{array}$ & $\begin{array}{l}0.406 \\
0.156 \\
0.125 \\
0.281 \\
0.062\end{array}$ \\
\hline $\begin{array}{l}332-38-1192 \\
28830598 \\
354-50-5899 \\
4131787 \\
7538695\end{array}$ & $\begin{array}{l}46 \\
47 \\
48 \\
49 \\
50\end{array}$ & $\begin{array}{l}0.187 \\
0.312 \\
0.312 \\
0.375 \\
1.125\end{array}$ & $\begin{array}{l}0.062 \\
0.093 \\
0.156 \\
0.031 \\
0.062\end{array}$ & $\begin{array}{l}0.062 \\
0.125 \\
0.093 \\
0.156 \\
0.593\end{array}$ & $\begin{array}{l}0.062 \\
0.093 \\
0.062 \\
0.187 \\
0.468\end{array}$ \\
\hline $\begin{array}{l}337-52-0095 \\
29519775 \\
323-46-8551 \\
29090351 \\
\text { XC } 1847 \quad 150\end{array}$ & $\begin{array}{l}51 \\
52 \\
53 \\
54 \\
55\end{array}$ & $\begin{array}{l}0.343 \\
1.562 \\
0.187 \\
0.5 \\
0.75\end{array}$ & $\begin{array}{l}0.187 \\
0.062 \\
0.062 \\
0.218 \\
0.093\end{array}$ & $\begin{array}{l}0.062 \\
0.531 \\
0.062 \\
0.093 \\
0.531\end{array}$ & $\begin{array}{l}0.093 \\
0.281 \\
0.062 \\
0.187 \\
0.125\end{array}$ \\
\hline $\begin{array}{l}\text { XC } 28946664 \\
12735864 \\
19032461 \\
26524054 \\
22791280\end{array}$ & $\begin{array}{l}56 \\
57 \\
58 \\
59 \\
60\end{array}$ & $\begin{array}{l}0.875 \\
0.468 \\
0.25 \\
0.656 \\
0.531\end{array}$ & $\begin{array}{l}0.062 \\
0.062 \\
0.031 \\
0.218 \\
0.062\end{array}$ & $\begin{array}{l}0.562 \\
0.312 \\
0.156 \\
0.187 \\
0.343\end{array}$ & $\begin{array}{l}0.25 \\
0.093 \\
0.062 \\
0.25 \\
0.125\end{array}$ \\
\hline Mean total & & 0.716 & 0.096 & 0.451 & 0.155 \\
\hline $\begin{array}{l}\text { Std. daviation } \\
\text { total }\end{array}$ & & 0.691 & 0.076 & 0.636 & 0.103 \\
\hline
\end{tabular}


APPENDIX E:

STATISTICS ON CULLING 
APPENDIX E:

STATISTICS ON CULLING

\begin{tabular}{|c|c|c|c|c|}
\hline \multirow[b]{2}{*}{ Culler } & \multicolumn{2}{|c|}{$\begin{array}{c}\text { Document Pulled } \\
(\% \text { of total })\end{array}$} & \multicolumn{2}{|c|}{ Time Taken $(\mathrm{min})$} \\
\hline & Mean & Limits & Mean & Limits \\
\hline $\begin{array}{l}\text { Edwards } \\
\text { Rutledge } \\
\text { Stigler } \\
\text { Wheatley } \\
\text { Phillips } \\
\text { Wengerski }\end{array}$ & $\begin{array}{r}11.00 \\
33.06 \\
7.16 \\
27.71 \\
21.92 \\
5.06\end{array}$ & $\begin{array}{r}8.02-13.98 \\
25.27-40.85 \\
3.12-11.20 \\
23.55-31.87 \\
18.00-25.84 \\
1.73-8.39\end{array}$ & $\begin{array}{r}5.95 \\
13.65 \\
11.53 \\
6.75 \\
6.42 \\
4.59\end{array}$ & $\begin{array}{l}2.86-9.04 \\
5.77-21.53 \\
1.48-21.57 \\
4.61-8.88 \\
4.05-8.79 \\
2.39-6.79\end{array}$ \\
\hline
\end{tabular}


APPENDIX E:

SPREADSHEET ON CLAIM FOLDER STATISTICS BY CULLER 


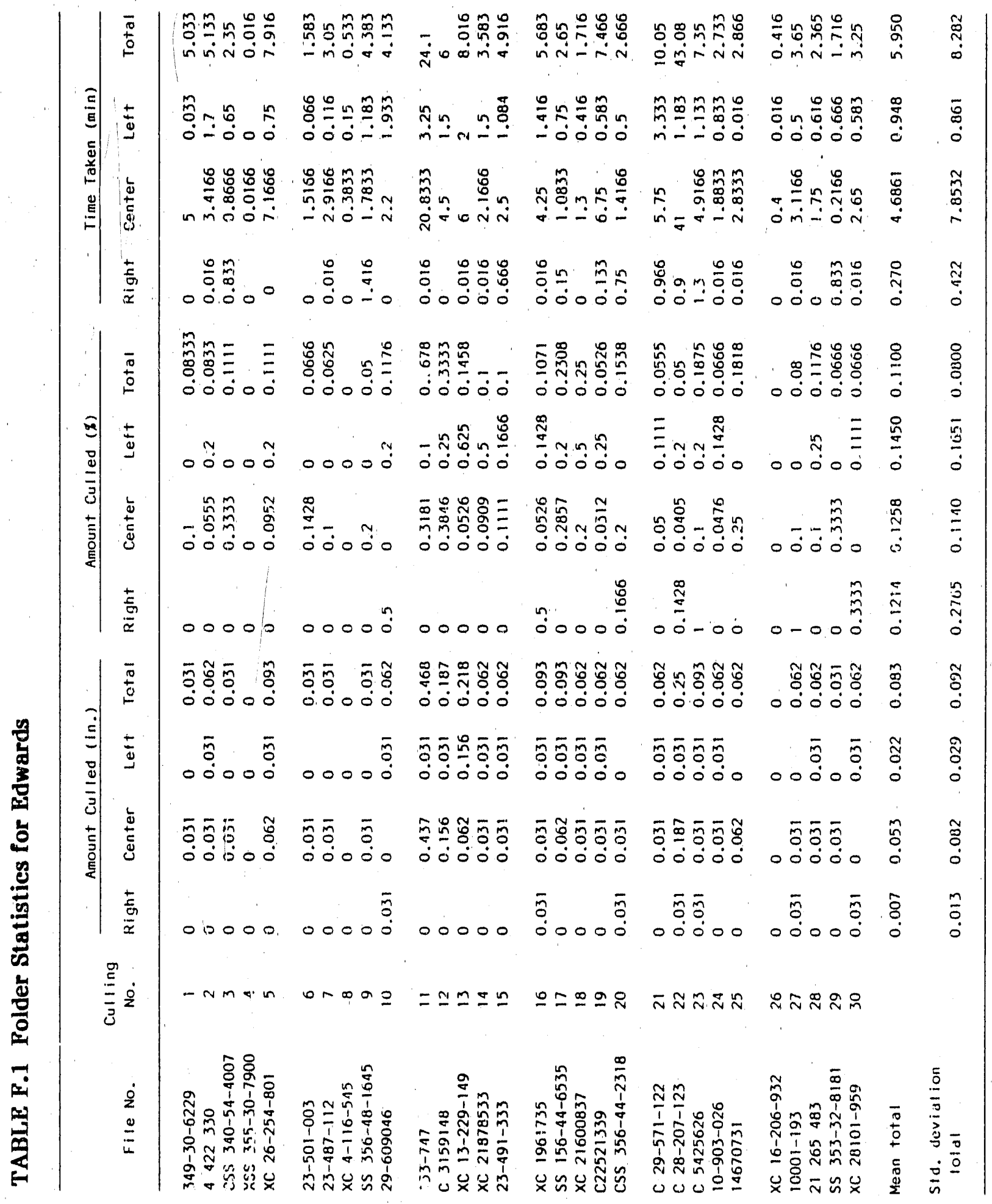




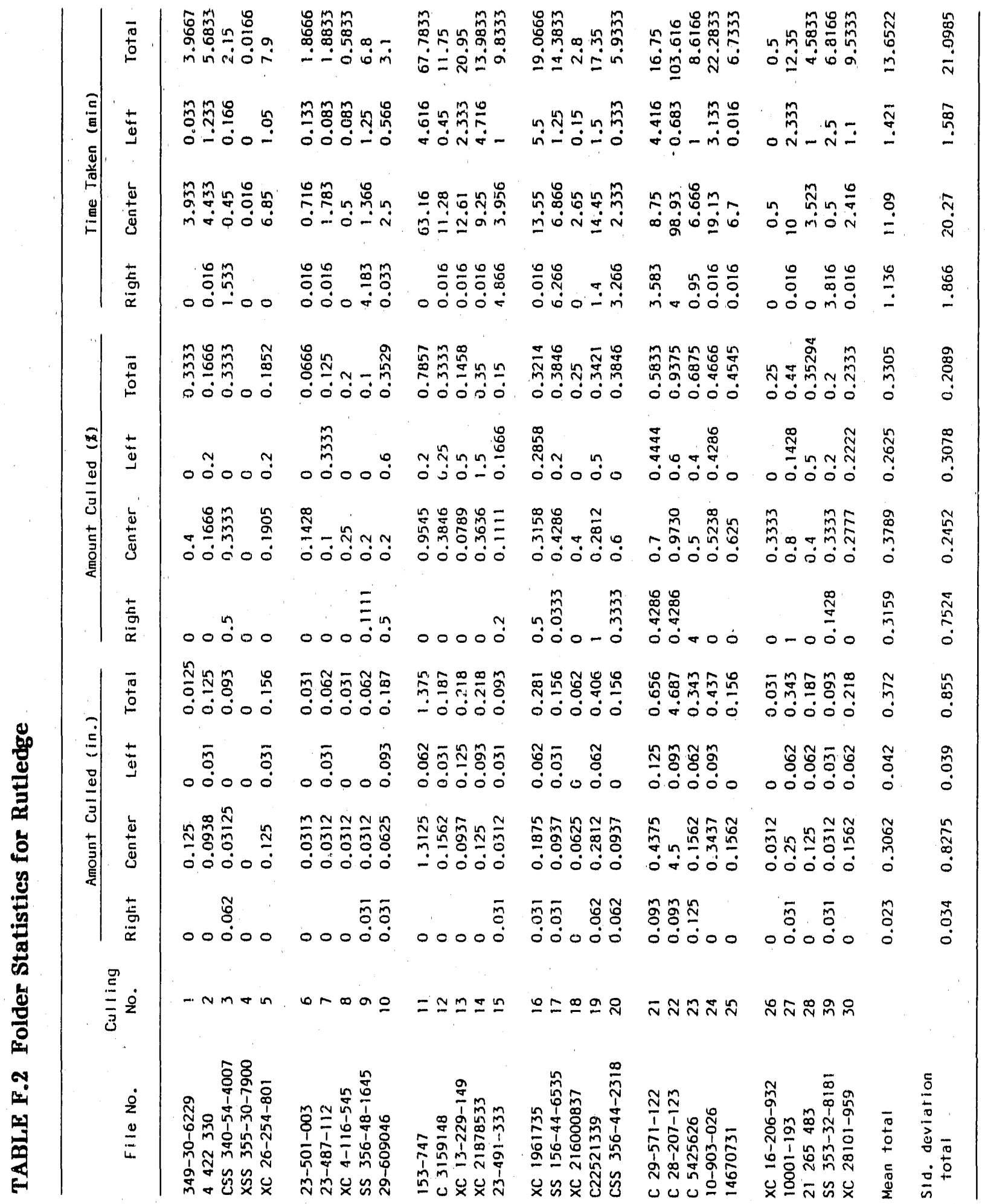




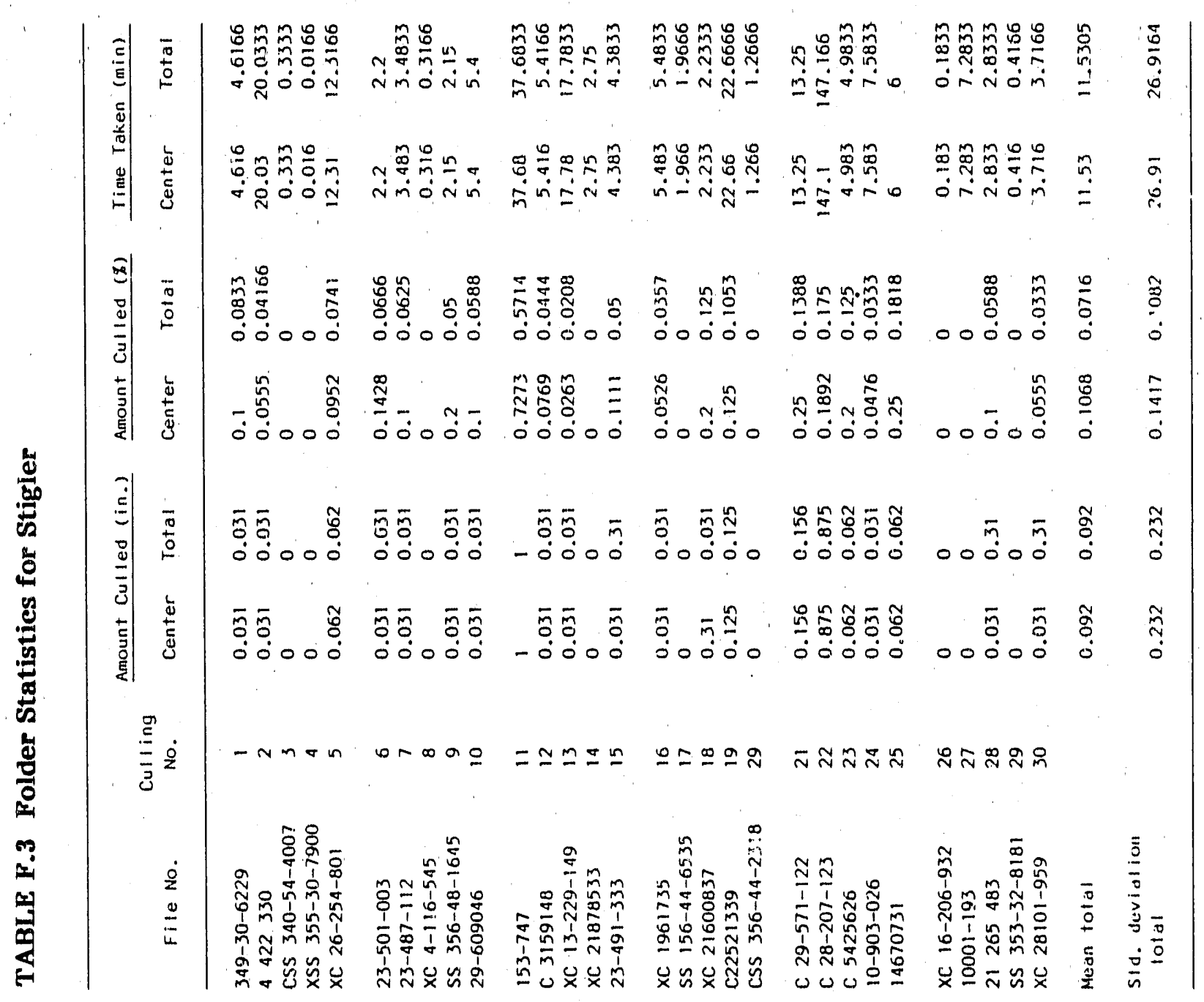




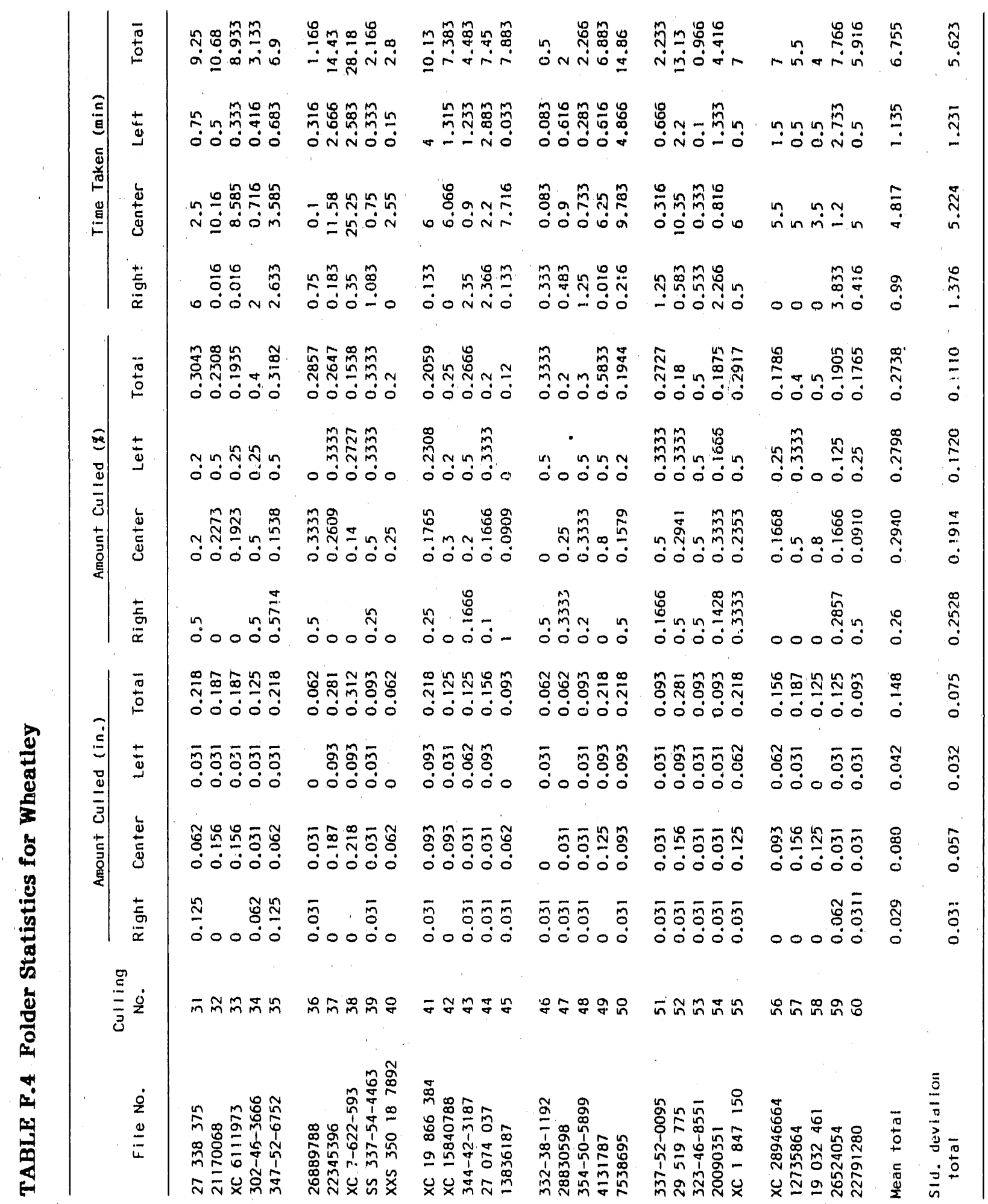




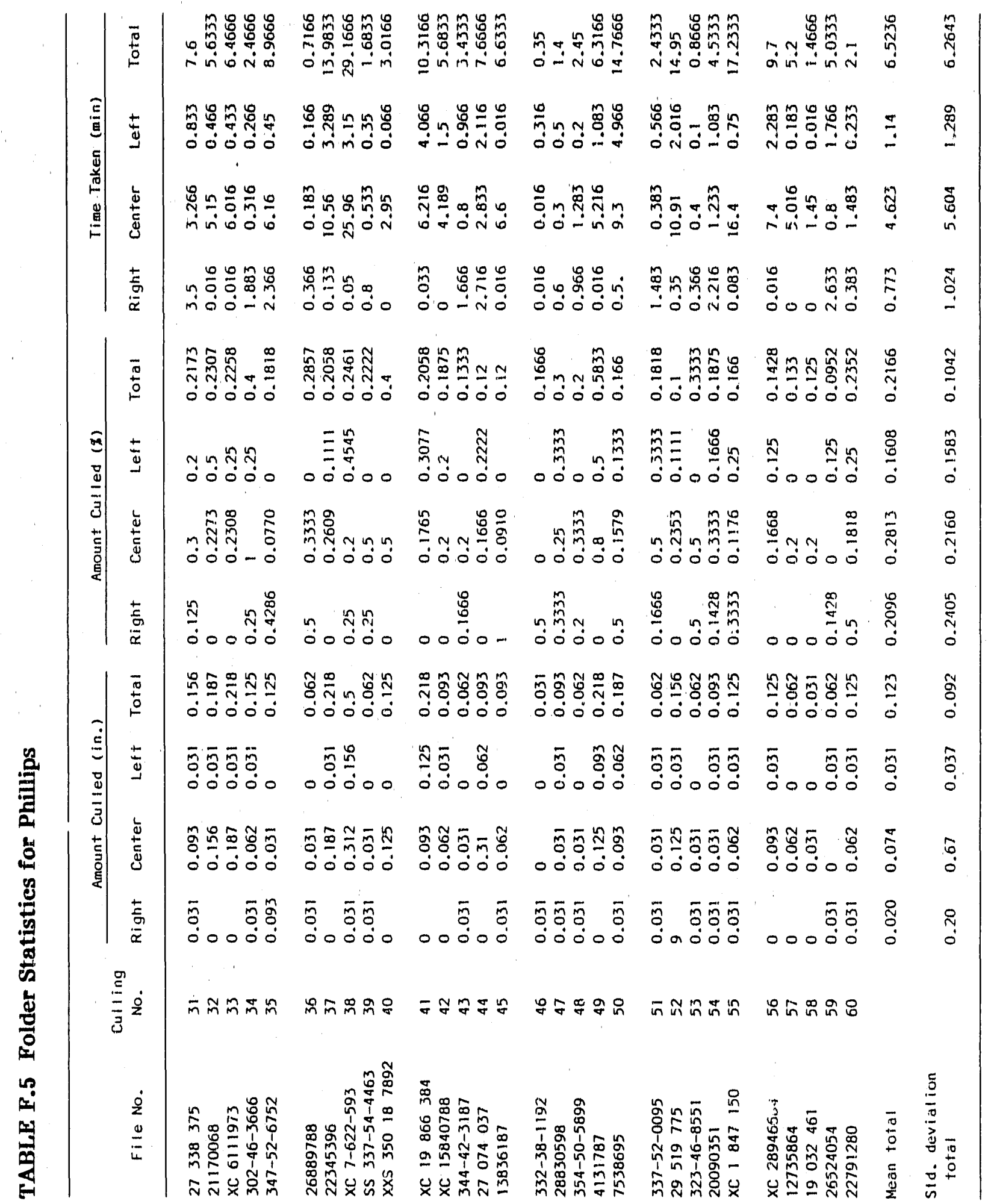




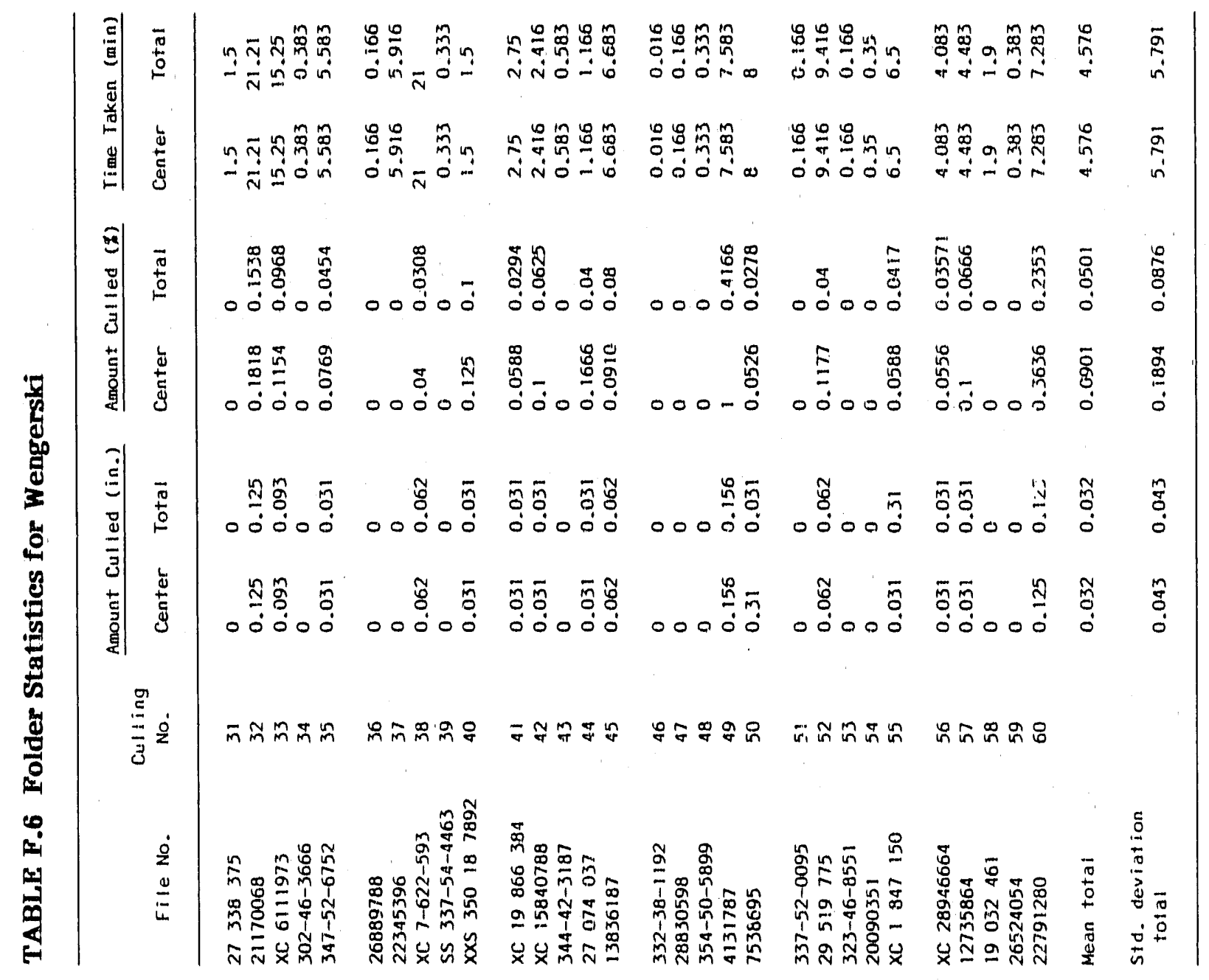


APPENDIX G:

LIST OF DOCUMENT'S REMOVED DURING CULLING 


\section{APPENDIX Gs}

\section{LIST OF DOCUMENTS REMOVED DURING CULLING}

G.1 Doouments removed by SImmons but not by uther cullers

DD 214

VA $07-3101$

VA $3-3177$

VA $4-.1833$

VA $4-5280$

VA $4-8268$

VA $8-93$

VA $8-2507$

VA $8-4600$

VA $8-564 \mathrm{C}$

VA 8-576A

VA $8-638 \mathrm{~A}$

VA $9-40-B$

VA $9-887 \mathrm{~A}$

VA $9-8888$

VA $10 \cdots 1349$

VA 10-2381

VA $10-2593$

VA 10-2652

VA $10-2731$

VA $10-2743$

VA 10-2827

VA $10-2829$

VA $10-7131$

VA $20-6560$

VA $20-6760$

VA 20-6889

VA 20-8270

VA 20-8271

VA 20-8332-2

VA 20-8992

VA 20-8993

VA $21 \mathrm{E}-1994$

VA 21E-1997"

VA $21 \mathrm{E}-8349$

VA 21-0515

VA 21-2507

VA $21-4100$

VA 21-4138

VA 21-4142
Request for information

Request for information on previous clalm

Document on veteran's indebtedness

Request for documentation for over payment

Fiscal code sheet

Award for burlal/transportation of veteran's body

Request for physical exam

Disability/compensation/pensiun code sheet

Deferred rating sheet

Request for medical information

Submission - rating decision

Transmittal of VA form

Notice of action taken on deductions from VA beneflt payments

Authorization for deduction

Social service report and sum mary

Power of attorney agreement

Record of hospitalization

Recelpt for remains and personal effects

Request for administrative/adjudicative action

Report - serlous illness

Application for outpatient treatment

Telephorie authorization

Exchange/beneflolary information request for

administrative/adjudicative action

Notice of benefit payment

Notice of benefit payment transaction

Correction cude sheet

C\&P master record audit write out

Notice of acceptance - C\&P input transaction

Award letter notification

Letter to veteran requesting information

Letter stating claim cannot be granted

Determination of basic ellgibility/entitlement

Education award

Deposition of Chapter 34

Improve pension eligibility report

Request for physical exam

Statement of income/net worth

Statement in support of claim

Authority/release of information 
VA 21-4180 Copy of request for certlflation by Soolal Seourlty and Rallroad Retirement

VA 21-4182 Applleation for dependenay/Indemnlty compensation

VA $21-4180$ Report on Income/property/business

VA $21-6164 \mathrm{E}$ Deforred clalm rating sheet

VA 21-626E Veteran's applloation for compensation

VA $21-684 D$ Dental rating sheet

VA. 21-6745 Control notice

VA 21-6789 Rating decision - falled to report for exam

VA 21-6796-1 Rating decision - continuation

VA 21-6798

VA $21-6798 \mathrm{D}$ Abllity/dlsabllity olalm

VA. $21-6880$

Death award

VA $21-686 \mathrm{C}$

Stop order/suspend payment notlce

VA 21-8332A-2 Declaration of dependent status

VA. 21-8947 Copy of an award letter

VA. 22-192K $C \& P$ award sheet

VA $22-1991$

VA counselling record

VA $22-1994 \mathrm{~A}$

Re-enrollment certlfication

VA 22-1995

VA 22-1997

Determination of basio eliglbility/entitlement

VA 22-19975-1

Request for change of program/training place

Education award

VA $22 \cdots 1998 \mathrm{~B}$

Education award code sheet

VA $22-1999 b$

Approval information of sredit/NON NCD Lrogram

VA $22-1999$

Notice - change in student status

VA $22-1999-1$

Enrollment certlficate

VA $22-8945$

Reerirollment certiflcate

VA $24-6694$

Education award

VA 24-706

VA $26-1817$

Recelvable/deduction transaction code sheet

Refund worksheet

VA $28-1923$

VA 28-8861

VA $45-851$

VA 119

VA 2322

Request for determination of loan guarantee

Declaration of marital/dependent status

Request for medical service

Insurance statement

Report of contact

VA 3102

VA 3230

VA 3232

VA 33542

Appointment/service organlzation clalmant representative

Request for information form

Reference slip

General information request

Authorization report

VA 3664

Notice - death of veteran

VA 482,68

Physical payment and adjustment code sheet

VA $686 \mathrm{C}$

Decoration of merit status

VA 8521

Stop/suspend payment notice

VA 8564

Rating sheet

IBM card

Admission record

Hospital record

Application for out-patient treatment 
Service medloal reoords

Medioal record

Clinioal reoord

Supplemental report

Dental oharts

Dental rating sheet

Drop flle letter to veteran - need records

Power of attorney

Physiolans affldavit

Letter from veteran

Letter to dootor

Letter from VA to veteran

Letter from National Service offloer from DAV

Letter to veteran's wife authorizing payment

Letter to clalms appeal board

Letter to adjustiment officer

Letter to attorney

Letter to senator

Letter to representative of state

Letter from senator

Letter from Disabled American Veterans

Letter from Department of Personnel in Springfield, Illinols

Letter to VFW

Letter" to Ameriogin Legion

Letter from National Service

Letter to Marine Corps

Letter to VA from Marino Corps

Letter from Accounts Rocivable

Letter from Insurance company

Letter frorn college attended/ing

Letter from dean of students

Report of location

Notice of change/student status

Education institution award - computer printout

Copy of cashiers sheck

Application for burial flag

Medical certifictate of death

Copy of certifliate of death

Funerel home till

Record of furiaral

Baptismal certificate

Certiflcate of life/birth

Declaration of marital status

Marriage certifloate

Marrlage license

Marriage certificate certification

Divorce paper

Divorce certlficate 
Divorce decree

Army Reserve status verification

Certificate of milltary service

Service information

Record of inllitary service

Copy of honorable discharge paper's

Target folder request

CARS master record printout

Computer printout sheet

Request for administrative/adjudicative action

Plece of paper with attachments (medlual records)

Envelope with dental x-rays

Memorandum from flnanolal offlcer

Memorandum on payment suspended

Note of income

Request for address Information

Change of address

Certificate of pension

Notice of pension

Continuation of pension

Stop payment notice

Memo of payment

Recelpts (computer printouts, slips, and register recelpts)

Eligibility/verification recelpt computer printout

Copy of authorization for direct deposit

Direct deposit form

Request for application to claim

Folder holder

Insurance from VA in Phlladelphia

Insurance award sheet

Data entry sheet

Release of information from VA

Consent to relesse information of records

Notice of assignment of claim number

Wisconsin Veteran Medical Service Agency - medical information

Departinent of Wisconsin - checklist letter

Illinois disability release form

Application for voucher

Application for vocational rehabilitation

Vocational rehabilitation revolving fund

Fiscal code sheet

Adjustment code sheet

Input transaction data sheet - notice of acceptance

Report denoting Hotchkins disease

Questionnaire 
G.2 Documents not removed by Simmons but removed by other cullers

VA 4-5234 Overpayment flash

VA 10-7131 Exchange of beneficiary information

VA 20-8332A-2 Letter to veteran

VA 20-8955. Letter to veteran

VA 20-8956 Letter to veteran

VA 20-8900 Letter to veteran on notice of overpayment or withheld benafits

VA 20-8993 Letter to veteran

VA 21E-1993 Enrollment certificate

VA 21E-1997 Education award

VA 21-074 Request for approval - school attendance

VA 21-4138 Statement in support of claim

VA 21-4189 Award/disallow of burial allow

VA 21-6789 Deferred/ confirmed rating decision

VA 21-6798 Award/disallowance disability claim

VA 21-8046 Payment notice

VA 21-8332A-2 Letter to veteran

VA 22-1997S

VA 22-1998B

VA 22-1999-1

VA $23-6538$

VA $28-8993$

Educational code sheet

Notice of change in student status or institutional course

Reenrollment certificate

Number and control record - name of current orphan

VA 2322

VA 2322

Letter to veteran

Appointment of Veteran Service Organization

Power of attorney

VA 3229

Routing slip between C21 (VA 21-4185 report/income/property/ business) and C22 (VA 8-4182 dependency/indemnity death)

VA $686 \mathrm{C}$

Decoration of merit status

Letter from veteran to VA

Letter to veteran

Letter from Social Security

Letter to veteran from Treasury Department

Letter from veteran's wife

Letter from doctor

Letter to doctor

Confidential Social Security benefit information

Award letter control document

$C \& P$ award computer printout

Education institution award computer printout

Eligibility verification computer printout

Stop, suspend, or resume computer printout

Telex computer printout

C\&P master record status master record sheet

Supplemental C\&P code sheet

Election of improved pension award

$C \& P$ disallowance code sheet

Certificate of eligibility

Certifiegte of degth 
Coroner's certificate of death

Marriage certificate

Birth certificate

Medical information

Medical notes

Medical records

Clinical record

Service medical records

Medical report history

Envelope containing medical records

Physical exam report

Report of physical exam

Physician statement

Affidavit from attending physician

Memorandum of a phone call

Honorable discharge

Request for army information

Request for information

Notice of claim folder removed

Target C\&P award

Target sheet

Target query

Scrap piece of paper

Reference note

Receipt

Rating decision

Rating sheet

Photostat of income records

War risk insurance photostat

World risk insurance

Order of transportation

Employment statement

Computer punch card

Piece of paper with Railroad Retirement Board on it

Folder paper 
$63 / 64$

APPENDIX H:

CALCULATION TABLES 


\section{APPENDIX H:}

\section{CALCULATION TABLES}

\section{H.1 Calculations for Edwards}

\begin{tabular}{lrr}
\hline \multirow{2}{*}{$\begin{array}{l}\text { Simmons's } \\
\text { Decision }\end{array}$} & \multicolumn{2}{c}{ Edwards's Decision } \\
\cline { 2 - 3 } & No & Yes \\
Yes & $\begin{array}{r}794 \\
(888.2)\end{array}$ & $(77.8)$ \\
No & 1296 & 11 \\
& $(1201.8)$ & $(105.2)$ \\
\hline
\end{tabular}

\begin{tabular}{lrrrrr}
\hline Cel1. & fo & fe & fo-fe & $($ fo-fe) & (fo- $f e)^{2} / f e$ \\
\hline A & 794 & 888.2 & -94.2 & 8873.64 & 9.991 \\
B & 172 & 77.8 & 94.2 & 8873.64 & 114.057 \\
C & 1296 & 1201.8 & 94.2 & 8873.64 & 7.384 \\
D & 11 & 105.2 & -94.2 & 8873.64 & 84.350 \\
Total & 2273 & 2273.0 & & & 215.782 \\
\hline
\end{tabular}

\begin{tabular}{lrr}
\hline Cell & \multicolumn{1}{c}{ fo } & \multicolumn{1}{c}{ fo ${ }^{2} / \mathrm{fe}$} \\
\hline A & 630436 & 709.791 \\
B & 29584 & 380.257 \\
C & $1,679,616$ & 1397.584 \\
D & 121 & 1.150 \\
Total & & 2488.782 \\
& &
\end{tabular}




\section{H.2 Calculations for Rutledge}

\begin{tabular}{lcc}
\hline \multirow{2}{*}{$\begin{array}{l}\text { Simmons's } \\
\text { Decision }\end{array}$} & \multicolumn{2}{c}{ Rutledge's Decision } \\
\cline { 2 - 3 } & No & Yes \\
\hline Yes & 305 & 661 \\
& $(633)$ & $(303)$ \\
No & 1253 & 51 \\
& $(895)$ & $(409)$ \\
\hline
\end{tabular}

\begin{tabular}{lrrrrr}
\hline Cell & fo & fe & fo-fe & $(\text { fo-fe })^{2}$ & $(f \circ-f e)^{2} / f e$ \\
\hline A & 305 & 663.0 & -358 & 128164.00 & 193.309 \\
B & 661 & 303.0 & 358 & 128164.00 & 422.984 \\
C & 1253 & 895.0 & 358 & 128164.00 & 143.200 \\
D & 51 & 409.0 & -358 & 128164.00 & 313.359 \\
Total & 2270 & 2270.0 & & & 1072.852 \\
\hline
\end{tabular}

\begin{tabular}{lrr}
\hline Cell & \multicolumn{1}{c}{ fo $^{2}$} & \multicolumn{1}{c}{ fo $^{2} / \mathrm{fe}$} \\
\hline A & 93025 & 140.309 \\
B & 436921 & 1441.984 \\
C & $1,570,009$ & 1754.20 \\
D & 2601 & 6.359 \\
Total & & 3342.852 \\
\hline
\end{tabular}




\section{H.3 Calculations for Stigler}

\begin{tabular}{lcc}
\hline $\begin{array}{l}\text { Simmons's } \\
\text { Decision }\end{array}$ & \multicolumn{1}{c}{ Stigler's Decision } \\
\hline Yes & $\begin{array}{c}707 \\
(734.9)\end{array}$ & $(34.1)$ \\
& Yes \\
No & 1041 & 19 \\
& $(1013.1)$ & $(46.9)$ \\
\hline
\end{tabular}

\begin{tabular}{lrrrcc}
\hline Cell & fo & fe & fo-fe & $(\text { fo-fe })^{2}$ & $(\text { fo }-f e)^{2} / f e$ \\
\hline A & 707 & 734.9 & -27.9 & 778.41 & 1.059 \\
B & 62 & 34.1 & 27.9 & 778.41 & 22.827 \\
C & 1041 & 1013.1 & 27.9 & 778.41 & 0.768 \\
D & 19 & 46.9 & -27.9 & 778.41 & 16.597 \\
Total & 1829 & 1829 & & & 41.251 \\
& & & & & \\
\hline
\end{tabular}

\begin{tabular}{rrr}
\hline Cel1 & \multicolumn{1}{c}{ fo $^{2}$} & \multicolumn{1}{c}{$\mathrm{fo}^{2} / \mathrm{fe}$} \\
\hline A & 499,849 & 680.159 \\
B & 3844 & 112.727 \\
C & $1,083,681$ & 1069.668 \\
D & 361 & 7.697 \\
Total & & 1870.251 \\
\hline
\end{tabular}




\section{H.4 Calculations for Wheatiey}

\begin{tabular}{lrr}
\hline & Wheatley's Decision \\
\cline { 2 - 3 } Simmons's & No & Yes \\
\hline Yes & $\begin{array}{r}529 \\
(714.4)\end{array}$ & $\begin{array}{c}414 \\
\text { No }\end{array}$ \\
No & $1031.6)$ & 94 \\
& $(848.6)$ & $(276.4)$ \\
\hline
\end{tabular}

\begin{tabular}{lrrrrr}
\hline Cell & fo & fe & fo-fe & (fo-fe) & (fo-fe $)^{2} / f e$ \\
\hline A & 529 & 711.4 & -182.4 & 33269.76 & 46.767 \\
B & 414 & 231.6 & 182.4 & 33269.76 & 143.652 \\
C & 1031 & 848.6 & 182.4 & 33269.76 & 39.206 \\
D & 94 & 276.4 & -182.4 & 33269.76 & 120.368 \\
Total & 2067 & 2067.0 & & & 349.993 \\
\end{tabular}

\begin{tabular}{lrr}
\hline Cell & \multicolumn{1}{c}{ fo $^{2}$} & \multicolumn{1}{c}{ fo $^{2} / \mathrm{fe}$} \\
\hline A & 279,841 & 393.367 \\
B & 171,396 & 740.052 \\
C & $1,062,961$ & 1252.606 \\
D & 8,836 & 31.968 \\
Total & & 2417.993 \\
\hline
\end{tabular}




\section{H.5 Calculations for Phillips}

\begin{tabular}{lrr}
\hline Simmons's & \multicolumn{2}{c}{ Phillips' Decision } \\
\cline { 2 - 3 } Decision & No & Yes \\
\hline Yes & 668 & 274 \\
No & $(808.5)$ & $(133.5)$ \\
& 1106 & 19 \\
& $(965.5)$ & $(159.5)$ \\
\hline
\end{tabular}

\begin{tabular}{lrrrrr}
\hline Cell & fo & fe & fo-fe & $($ fo-fe) & (fo-fe) $)^{2} / f e$ \\
& & & & & \\
A & 668 & 808.5 & -140.5 & 19740.25 & 24.415 \\
B & 274 & 133.5 & 140.5 & 19740.25 & 147.867 \\
C & 1106 & 965.5 & 140.5 & 19740.25 & 20.446 \\
D & 19 & 159.5 & -140.5 & 19740.25 & 123.763 \\
Total & 2067 & 2067.0 & & & 316.491 \\
\hline
\end{tabular}

\begin{tabular}{rrr}
\hline Cell & \multicolumn{1}{c}{ fo $^{2}$} & \multicolumn{1}{c}{ fo $^{2} / \mathrm{fe}$} \\
\hline A & 446,224 & 551.916 \\
B & 75,076 & 562.367 \\
C & $1,223,236$ & 1266.945 \\
D & 361 & 2.263 \\
Total & & 2383.491 \\
\hline
\end{tabular}




\section{H.6 Calculations for Wengerski}

\begin{tabular}{lcc}
\hline Simmons's & \multicolumn{2}{l}{ Wengerski's Decision } \\
\cline { 2 - 3 } Decision & No & Yes \\
\hline Yes & 555 & 25 \\
& $(563.7)$ & $(16.3)$ \\
No & 759 & 13 \\
& $(750.3)$ & $(21.7)$ \\
\hline
\end{tabular}

\begin{tabular}{lrrrcc}
\hline Cell & fo & fe & fo-fe & (fo-fe) ${ }^{2}$ & (fo- fe $)^{2} / f e$ \\
\hline A & 555 & 563.7 & -8.70 & 75.69 & 0.134 \\
B & 25 & 16.3 & 8.70 & 75.69 & 4.644 \\
C & 759 & 750.3 & 8.70 & 75.69 & 0.101 \\
D & 13 & 21.7 & -8.70 & 75.69 & 3.488 \\
Total & 1352 & 1352.0 & & & 8.367 \\
\hline
\end{tabular}

\begin{tabular}{lrr}
\hline Cel1 & \multicolumn{1}{c}{ fo ${ }^{2}$} & \multicolumn{1}{c}{ fo ${ }^{2} / \mathrm{fe}$} \\
\hline A & 308,025 & 564.434 \\
B & 625 & 38.344 \\
C & 576,081 & 767.801 \\
D & 169 & 7.788 \\
Total & & 1360.367 \\
\hline
\end{tabular}


$71 / 72$

APPENDIX I:

REGRESSION ANALYSIS 


\section{APPENDIX I:}

\section{REGRESSION ANALYSIS}

TABLE I.1 R Squared Table ${ }^{\mathrm{a}}$

\begin{tabular}{lcc}
\hline & $\begin{array}{c}\text { Dependent } \\
\text { Variable 1: } \\
\text { Culling Time }\end{array}$ & $\begin{array}{c}\text { Dependent } \\
\text { Vartable 2 } \\
\text { Percentage } \\
\text { Removed }\end{array}$ \\
\hline Edwards & 0.900 & 0.001 \\
Rutiedge & 0.873 & 0.404 \\
Stigler & 0.970 & 0.090 \\
Wheatley & 0.887 & $0.286^{\circ}$ \\
Philips & 0.831 & 0.082 \\
Wengerski & 0.714 & 0.000 \\
\hline &
\end{tabular}




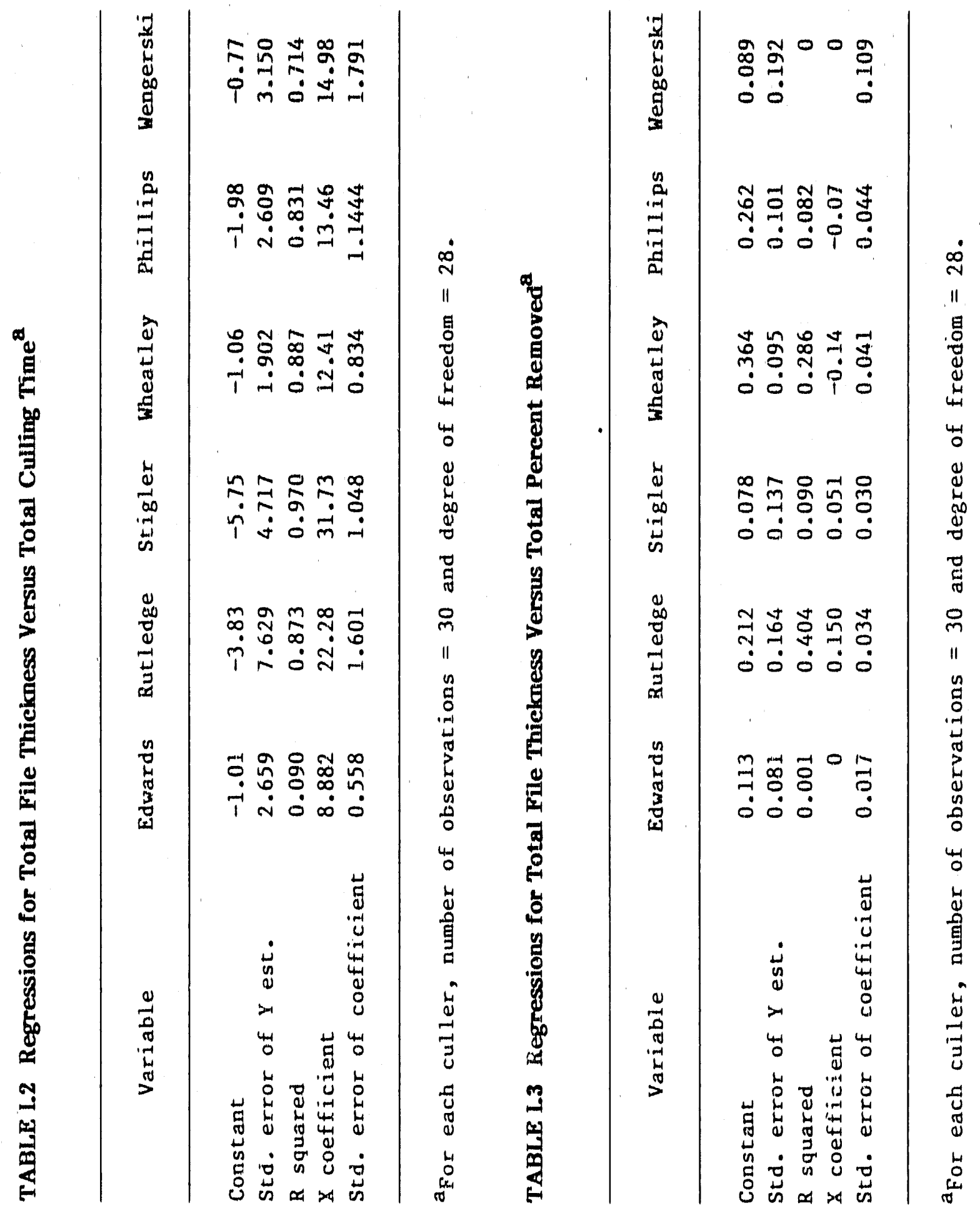



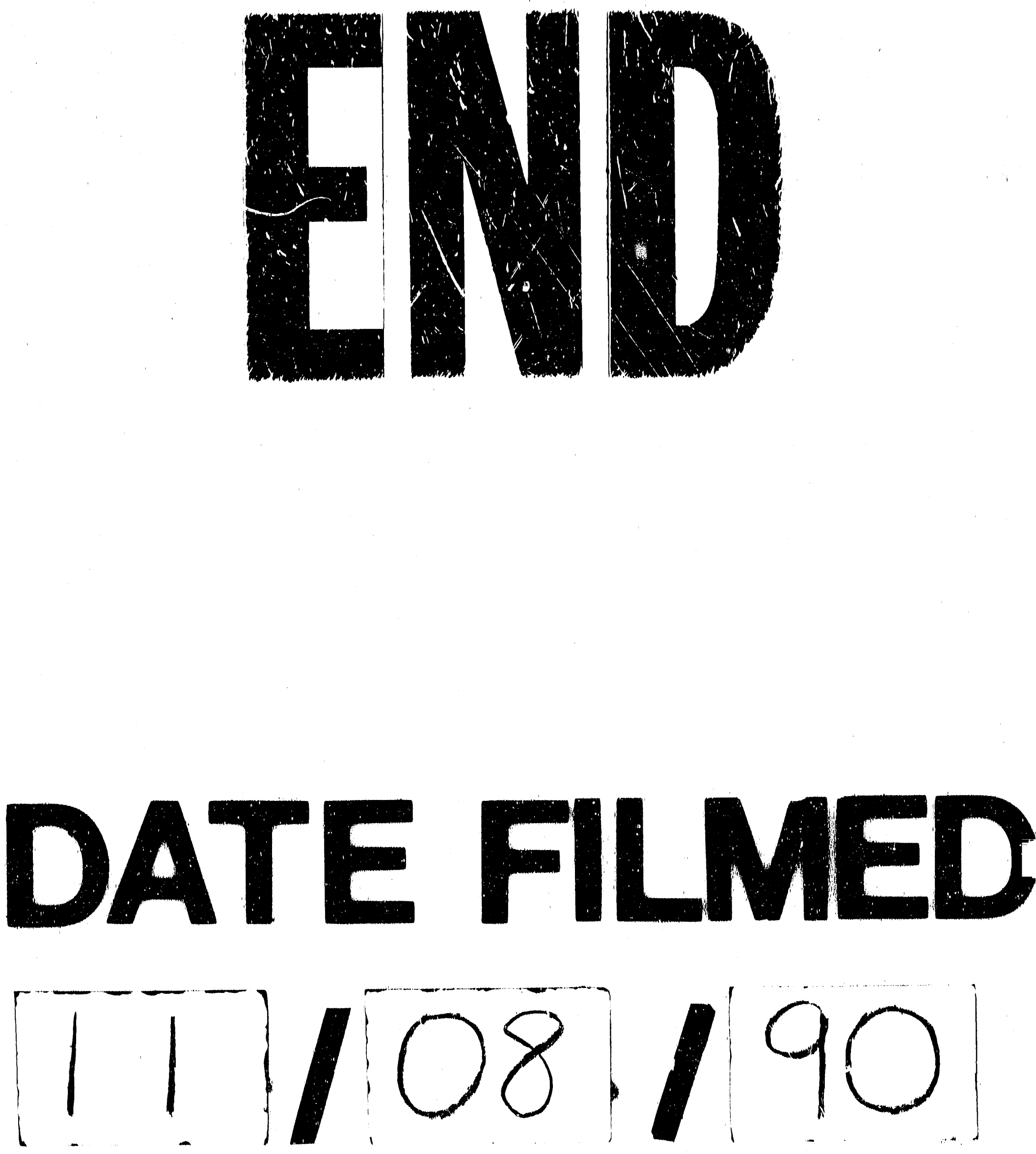
